\title{
Matrix metalloproteinase-9 deficiency protects mice from severe influenza $A$ viral infection
}

\author{
Joselyn Rojas-Quintero, ${ }^{1}$ Xiaoyun Wang, ${ }^{1}$ Jennifer Tipper, ${ }^{2}$ Patrick R. Burkett, ${ }^{1}$ Joaquin Zuñiga, ${ }^{3}$ \\ Amit R. Ashtekar, ${ }^{2}$ Francesca Polverino, ${ }^{1,4}$ Amit Rout, ${ }^{1}$ llyas Yambayev, ${ }^{1}$ Carmen Hernández, ${ }^{3,5}$ \\ Luis Jimenez, ${ }^{3}$ Gustavo Ramírez, ${ }^{3}$ Kevin S. Harrod, ${ }^{2}$ and Caroline A. Owen ${ }^{1,4}$ \\ 'Division of Pulmonary and Critical Care Medicine, Brigham and Women's Hospital, and Harvard Medical School, Boston, \\ Massachusetts, USA. ${ }^{2}$ Department of Anesthesiology and Perioperative Medicine, Division of Molecular and Translational \\ Biomedicine, School of Medicine, University of Alabama-Birmingham, Birmingham, Alabama, USA. ${ }^{3}$ Laboratory of \\ Immunobiology and Genetics, and Intensive Care Unit, Instituto Nacional de Enfermedades Respiratorias Ismael Cosío \\ Villegas, Mexico City, Mexico. ${ }^{4}$ Lovelace Respiratory Research Institute, Albuquerque, New Mexico, USA. ${ }^{5}$ Escuela de \\ Medicina y Ciencias de la Salud, Tecnologico de Monterrey, Mexico City, Mexico.
}

Matrix metalloproteinase-9 (MMP-9) cleaves various proteins to regulate inflammatory and injury responses. However, MMP-9's activities during influenza A viral (IAV) infections are incompletely understood. Herein, plasma MMP-9 levels were increased in patients with pandemic H1N1 and seasonal IAV infections. MMP-9 lung levels were increased and localized to airway epithelial cells and leukocytes in H1N1-infected WT murine lungs. H1N1-infected Mmp-9-/- mice had lower mortality rates, reduced weight loss, lower lung viral titers, and reduced lung injury, along with lower E-cadherin shedding in bronchoalveolar lavage fluid (BALF) samples than WT mice. H1N1-infected $\mathrm{Mmp}-\mathrm{9}^{-/-}$mice had an altered immune response to IAV with lower BALF PMN and macrophage counts, higher Th1-like $\mathrm{CD4}^{+}$and $\mathrm{CD8}^{+} \mathrm{T}$ cell subsets, lower T regulatory cell counts, reduced lung type I interferon levels, and higher lung interferon- $\gamma$ levels. Mmp- 9 bone marrowchimera studies revealed that Mmp-9 deficiency in lung parenchymal cells protected mice from IAV-induced mortality. H1N1-infected Mmp-9-/- lung epithelial cells had lower viral titers than H1N1-infected WT cells in vitro. Thus, H1N1-infected $M$ mp-9-/- mice are protected from IAV-induced lung disease due to a more effective adaptive immune response to IAV and reduced epithelial barrier injury due partly to reduced E-cadherin shedding. Thus, we believe that MMP-9 is a novel therapeutic target for IAV infections.

Authorship note: JRQ, XW, and JT contributed equally to this work. KSH and $\mathrm{CAO}$ are $\mathrm{CO}-$ senior authors.

Conflict of interest: The authors have declared that no conflict of interest exists.

License: Copyright 2018, American Society for Clinical Investigation.

Submitted: December 5, 2017 Accepted: November 6, 2018 Published: December 20, 2018

\section{Reference information:} JCI Insight. 2018;3(24):e99022 https://doi.org/10.1172/jci. insight. 99022 .

\section{Introduction}

Seasonal influenza epidemics cause $0.25-0.5$ million deaths/year worldwide and mortality increases significantly in pandemic years (1). Influenza A viral (IAV) infections are associated with a high healthcare burden especially among the elderly, infants, immunosuppressed individuals, and pregnant women who are at increased risk of developing multiorgan failure (2, 3). Approximately $44.8 \%$ of individuals 19 years of age or older were vaccinated against IAV infection in the 2014-2015 season (4), representing only a $1.6 \%$ increase in coverage from the 2013-2014 season (4). Additionally, not all subjects develop robust protective immune responses to these vaccines $(5,6)$. Antiviral drugs (e.g., oseltamivir) do not prevent IAV-associated mortality in all individuals, especially when there is a delay in initiating therapy $(7,8)$. Thus, there is an urgent need to develop more effective therapies that limit the high healthcare burden associated with IAV infections.

Matrix metalloproteinases (MMPs) are a family of approximately 24 zinc-dependent metalloproteinases. They are characterized by a multidomain structure that includes (a) a prodomain at the amino terminus, (b) a catalytic domain containing the active-site zinc atom, and (c) a carboxy-terminal hemopexin domain that can bind MMPs to susceptible substrates. MMPs regulate tissue remodeling associated with morphogenesis and tissue repair; inflammatory responses to injury; the proliferation, migration, differentiation, and apoptosis of cells; angiogenesis; and host defense (9). 
Table 1. Demographic and clinical characteristics of the human cohort

\begin{tabular}{|c|c|c|c|c|}
\hline $\begin{array}{l}\text { Characteristics of the } \\
\text { human cohort }\end{array}$ & $\begin{array}{l}\text { Uninfected Healthy Volunteers } \\
\qquad(N=14)\end{array}$ & $\begin{array}{l}\text { Seasonal IAV Infection } \\
\qquad(N=10)\end{array}$ & H1N1 IAV Infection $(N=66)$ & $P$ value \\
\hline Males $(\%)^{A}$ & $8(57)$ & $6(60)$ & $33(66)$ & NS \\
\hline Influenza vaccination $n(\%)^{A}$ & $11(79)$ & $0(0)$ & $1(2)$ & $0.001^{c}$ \\
\hline Diabetes mellitus $n(\%)^{A}$ & $0(0)$ & $0(0)$ & $9(14)$ & NS \\
\hline Obese $n(\%)^{A, D}$ & $0(0)$ & $2(20)$ & $30(45)$ & $0.004^{\mathrm{E}}$ \\
\hline
\end{tabular}

Plasma samples from patients infected with seasonal IAV or 2009 pandemic H1N1 IAV. The samples from patients infected with seasonal influenza were obtained during the first 2 weeks of the onset of symptoms. For subjects infected with H1N1, the samples were obtained during the first 30 days after they were admitted to the intensive care unit. ${ }^{A}$ Categorical variables were analyzed with the Z-proportions test. Noncategorical data were analyzed using 1-way ANOVAs followed by Brown-Forsythe equal variance test. Pairwise comparisons for parametric data were performed using 2-sided Student's $t$ tests. ${ }^{B} A g e$

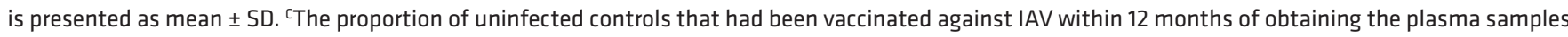
was greater than that of subjects infected with seasonal IAV and H1N1 $\left(P<0.001\right.$ for both comparisons). ${ }^{\circ}$ Obesity was defined as body mass index $>30 \mathrm{~kg} /$ $\mathrm{m}^{2}$. EThe proportion of obese subjects infected with $\mathrm{H} 1 \mathrm{~N} 1 \mathrm{IAV}$ was greater than that of uninfected obese subjects $(P=0.004)$. ${ }^{\mathrm{FPaO}} / 2 / \mathrm{FiO} \mathrm{O}_{2}$ ratio is a marker of the severity of acute lung injury/adult respiratory distress syndrome (22). Numbers in parentheses are the interquartile range. ${ }^{\mathrm{C} P l a s m a ~ M M P-9}$ levels were measured using an ELISA and data are presented as median (interquartile range). Data were analyzed using an ANOVA on ranks and pair-wise multiple comparisons using Dunn's method, followed by pair-wise comparison using Mann-Whitney $U$ tests. ${ }^{H} P<0.001$ for both seasonal IAV and H1N1 IAV versus uninfected controls. There was no significant correlation between plasma MMP-9 levels and the $\mathrm{PaO}_{2} / \mathrm{FiO}_{2}$ ratio using Pearson's correlation $(P=0.763)$. IAV, influenza A virus; NS, not significant.

MMP-9 (gelatinase B; type IV collagenase) is expressed by epithelial cells (10), endothelial cells (11), and all leukocytes $(12,13)$. With the exception of PMNs (13), cells need to be activated to express MMP-9 (12, 13). MMP-9 is secreted by cells in its latent pro-MMP form, and is then activated in the extracellular space by other proteinases and oxidants, which disrupt the interaction between the active site $\mathrm{Zn}^{2+}$ atom and a cysteine residue in the prodomain (the cysteine switch mechanism; see ref. 14). This process is followed by autolytic cleavage of part of the prodomain (12).

Little is known about the contributions of MMPs to IAV infections. In other models of disease, Mmp-9 cleaves and thereby activates/inactivates proinflammatory mediators to regulate lung inflammatory responses to allergens (15). MMP-9 also regulates acute lung injury $(16,17)$, disrupts airway epithelial barrier function (18), degrades a broad spectrum of extracellular matrix (ECM) proteins (13), and sheds E-cadherin from ovarian epithelial cell surfaces (19). MMP-9 activates latent growth factors and permogens that increase vascular permeability (20), and promotes abnormal distal airway epithelial repair in bleomycin-treated mice (21). Thus, we hypothesized that MMP-9 regulates lung inflammation and injury during IAV infections. To test this hypothesis, we measured MMP-9 levels in blood and/or lung samples from IAV-infected human subjects and mice. We compared survival rates, pulmonary inflammation, acute lung injury, and lung viral titers in WT versus $M m p-9^{-1-}$ mice and/or $M m p-9$ bone marrow-chimeric (BM-chimeric) mice. Our results show that MMP-9 expression is increased in IAV-infected human subjects and mice. In addition, $M m p^{-9^{-1-}}$ mice are protected from IAV-associated mortality likely due to their reduced lung injury, and their enhanced adaptive immune response to IAV infection leading to increased IAV clearance from the lungs. $M m p-9$ deficiency in lung parenchymal cells protects mice from IAV-induced mortality. Thus, our results identify MMP-9 as a potential therapeutic target for serious IAV infections.

\section{Results}

MMP-9 levels are increased in blood and/or lung samples from IAV-infected human subjects and mice. Demographic and clinical data on the human subjects studied are shown in Table 1. There were no significant differences between the groups in age, sex, or the percentage of subjects with diabetes mellitus. Human subjects with laboratory-confirmed seasonal IAV or A/California/07/2009 H1N1 infection had more than 20-fold higher plasma MMP-9 levels than uninfected control subjects (Figure 1A). Plasma MMP-9 levels did not correlate with the arterial oxygen tension/fractional inspired oxygen $\left(\mathrm{PaO}_{2} / \mathrm{FiO}_{2}\right)$ ratio, a measure of the severity of acute lung injury (22) (Table 1). 
A

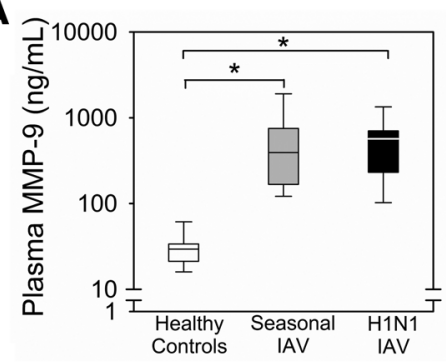

C

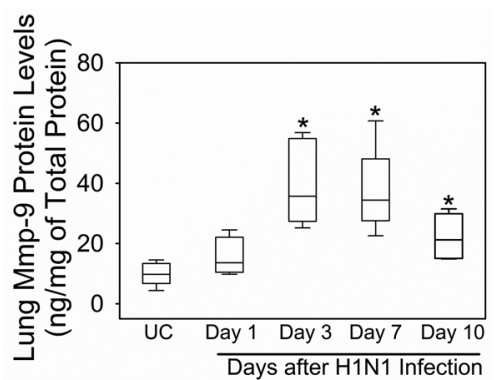

B

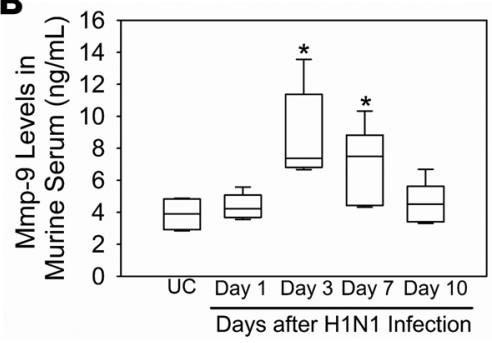

Figure 1. MMP-9 levels were increased in blood and/or lung samples from IAV-infected human subjects and WT mice. (A) MMP-9 protein levels were measured in plasma samples obtained from human patients diagnosed with A/ California/07/2009 H1N1 strain influenza infection $(n=66)$ or seasonal IAV infection $(n=10)$, or uninfected healthy control subjects ( $n=14)$ using an ELISA. For subjects infected with seasonal influenza, samples were obtained within the first 2 weeks of onset of symptoms. For subjects infected with H1N1, samples were obtained during the first 30 days after they were admitted to the intensive care unit. ${ }^{*} P<0.001$ versus the group indicated. (B) WT mice were infected an $\mathrm{LD}_{20}$ inoculum of $\mathrm{H1N} 1 \mathrm{IAV}$ by the intranasal route. Serum Mmp-9 levels were measured in infected mice on days 1-10 postinfection or uninfected control (UC) WT mice using an ELISA ( $n=5-7$ mice/group). ${ }^{*} P<0.05$ versus uninfected controls. (C) WT mice were infected with a $\mathrm{LD}_{20}$ inoculum by the intranasal route. At postinfection intervals, lungs were removed from infected mice or uninfected controls (UC) and Mmp-9 protein levels were measured using ELISA and normalized to total protein levels (4-5 mice/group). ${ }^{*} P<0.05$ versus uninfected controls. All box-and-whisker plots show medians and 25th and 75th percentiles, and the whiskers show the 10th and 90th percentiles. All data were analyzed with 1-way ANOVAs followed by pair-wise testing with Mann-Whitney $U$ tests.

C57BL/6 WT mice were infected with an $\mathrm{LD}_{20}$ inoculum of $\mathrm{H} 1 \mathrm{~N} 1$, and Mmp-9 levels were measured in serum and lung samples. Serum Mmp-9 levels were increased on days 3 and 7 postinfection (p.i.) (Figure 1B). Mmp-9 protein levels increased on day 3 p.i. in homogenates of lung samples from WT mice, remained elevated for 7 days, and were returning towards baseline levels by day 10 p.i. (Figure 1C). Double-immunostaining experiments performed on sections of lungs from uninfected versus H1N1-infected WT mice localized the cellular sources of Mmp-9 in the lungs. Uninfected WT mice had minimal or no Mmp-9 staining in their lungs (Figure 2A). Mmp-9 staining was increased in airway epithelial cells from day 7 to day 10 p.i. (Figure 2A). PMNs were not present in the airways of uninfected mice. Mmp- 9 staining was detected in PMNs recruited into the airways of IAV-infected WT mice (especially on day 10 p.i.; Figure 2B), as expected because PMNs store Mmp-9 protein within their gelatinase granules (13). Mmp-9 staining was induced in airway macrophages (Figure 2C) on days 7 and 10 p.i. Mmp-9 staining was observed in $\mathrm{CD}^{+} \mathrm{T}$ cells (Figure 2D) and B cells (Figure 2E) recruited into the airways on days 7 and 10 p.i. Staining for Mmp-9 was not detected in $\mathrm{CD} 8^{+} \mathrm{T}$ cells (Supplemental Figure 1; supplemental material available online with this article; https://doi.org/10.1172/jci.insight.99022DS1). No staining was detected in the lungs of infected WT mice stained with nonimmune primary antibodies (Figure 2).

Mmp-9-- mice are protected from H1N1-induced mortality, and have reduced lung viral titers and a reduced lung innate immune inflammatory reaction following $H 1 N 1$ infection. Mmp-9-/- mice infected with an $\mathrm{LD}_{80} \mathrm{H} 1 \mathrm{~N} 1$ inoculum had better survival rates (Figure 3A) and reduced loss of body weight from days 4-7 p.i. (Figure 3B) versus infected WT mice. Mmp-9-/ mice infected with an $\mathrm{LD}_{20} \mathrm{H} 1 \mathrm{~N} 1$ inoculum had reduced lung viral titers as assessed by quantitative real-time reverse-transcription PCR (qRT-PCR) on days 7-10 p.i. (Figure 4A) and multifoci staining assays on day 10 p.i. (Figure 4B).

H1N1-infected Mmp-9-- mice had reduced peribronchial inflammation in lung sections on days 7-10 p.i. compared with WT mice (Supplemental Figure 2). H1N1-infected $\mathrm{Mmp}^{-9^{-/}}$mice had lower bronchoalveolar 

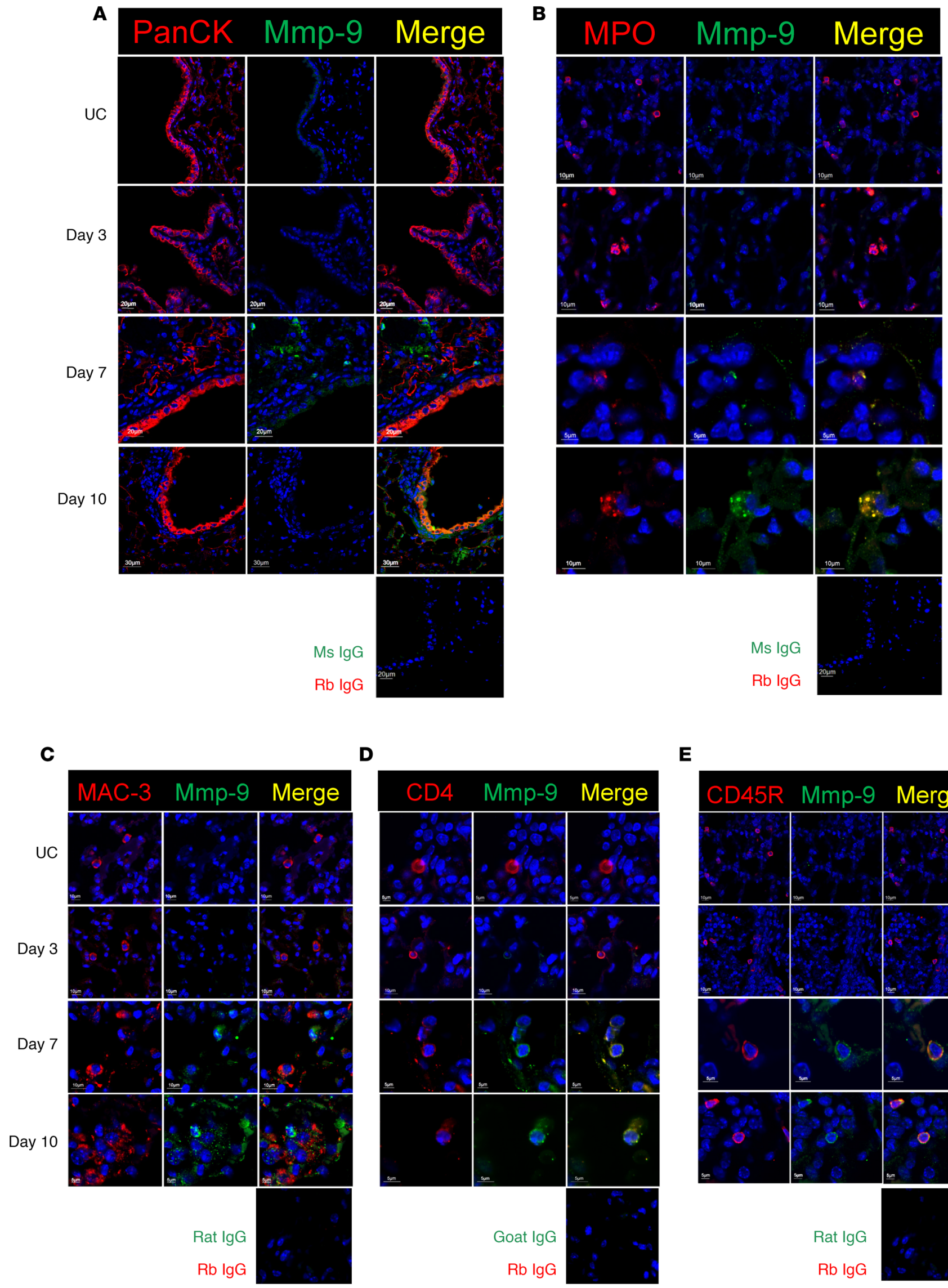

D

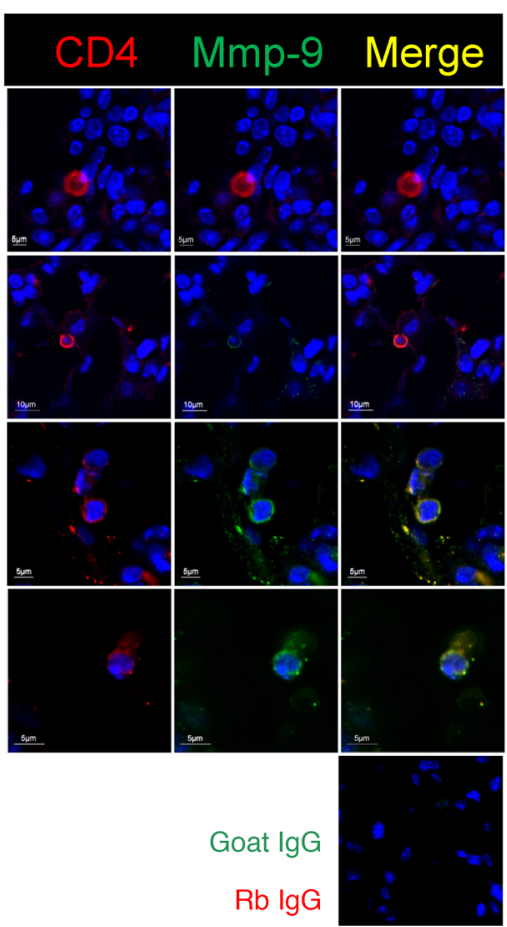

E

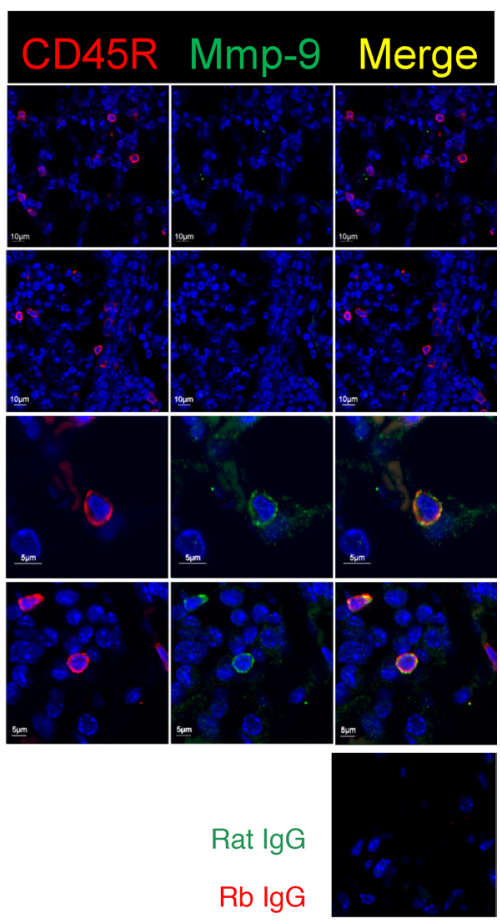


Figure 2. Mmp-9 staining was increased in airway epithelial cells and some leukocyte subsets in WT mice infected with H1N1 IAV. WT mice were infected by the intranasal route with an $\mathrm{LD}_{20}$ inoculum of $\mathrm{H} 1 \mathrm{~N} 1$ mice and lungs were inflated and removed on days 3,7 , and 10 after infection. Lung sections from H1N1-infected mice or uninfected control (UC) WT mice were double immunostained for Mmp-9 (in green) and for markers (in red) of lung epithelial cells (pancytokeratin [PanCK] in A), PMNs (myeloperoxidase [MPO] in B), macrophages (MAC-3 in C), CD4+ T cells (CD4 in D) and B cells (CD45R in E). The lungs of infected mice were also stained with nonimmune isotype-matched primary antibodies (rabbit [Rb] IgG, murine [Ms] IgG, rat lgG, or goat lgG], and images of these sections are shown in the lower panels of each subfigure. Nuclei were counterstained blue with 4',6-diamidino-2-phenylindole. Immunostained lung sections were examined with a confocal microscope and merged images are shown in the right panels. Images shown are representative of sections from 5 mice per experimental group. Original magnification, $\times 400$.

lavage fluid (BALF) total leukocyte counts on day 3 and 7 p.i. than infected WT mice (Figure 5A), which was mostly due to lower BALF macrophage counts on day 3 p.i. (Figure $5 \mathrm{~B}$ ) and lower BALF PMN counts on day 7 p.i. (Figure 5C)

H1N1-infected Mmp-9-1- mice have reduced lung levels of key proinflammatory mediators, reduced lung levels of type I interferons, but increased lung levels of interferon- $\gamma$. As MMP-9 cleaves cytokines and chemokines in vitro (9), lung levels of mediators that regulate the recruitment of myeloid leukocytes into the lung were compared in homogenates of lungs from H1N1-infected WT and Mmp-9-1- mice. Infected $M m p-9^{-/-}$mice had lower lung levels of Cxcl-2 on days 3-10 p.i. (Figure 6A), Cxcl-6 on days 3-7 p.i. (Figure 6B), Ccl-2 on day 3 p.i. (Figure 6C), Ccl-3 on day 3 p.i. (Figure 6D), Ccl-5 on day 7 p.i. (Figure 6E), Il-6 on days 3-10 p.i. (Figure 6F), G-csf on day 3 p.i. (Figure 6G), Tnf- $\alpha$ on day 10 p.i. (Figure 6H), and Tgf- $\beta$ on day 1 p.i. (Figure 6I). However, H1N1-infected $M m p^{-9^{--}}$mice had higher lung levels of Il-1 $\beta$ on day 1 p.i. (Supplemental Figure 3A). H1N1-infected WT and $M m p^{-9^{-1}}$ mice had similar lung levels of Cxcl-1 and antiinflammatory Il-10 (Supplemental Figure 3, B and C).

As the generation of antiviral interferons (IFNs) is an important determinant of outcomes after IAV infection, lung IFN levels were measured in homogenates of lungs from H1N1-infected WT and Mmp- $9^{-/-}$mice. Surprisingly, infected $M m p-9^{-1-}$ mice had lower lung levels of two type I IFNs (Ifn- $\alpha$ on day 3 p.i. [Figure 7A] and Ifn- $\beta$ on day 3 p.i. [Figure 7B]). However, infected $M m p-9^{-1-}$ mice had higher lung levels of Ifn- $\gamma$ on day 7 p.i. (Figure 7C).

H1N1-infected Mmp-9-1- mice have an enhanced pulmonary adaptive immune response. BALF lymphocyte counts were increased in H1N1-infected $\mathrm{Mmp}^{-9^{-/}}$mice on day 10 p.i. (Figure 5D). Lymphocyte subsets were quantified in enzymatic lung digests from H1N1-infected WT and $M m p-9^{-/-}$mice on days 3-10 p.i. using immunostaining and flow cytometry. Compared with H1N1-infected WT mice, infected $M m p-9^{-1}$ mice had higher $\mathrm{CD}^{+} \mathrm{T}$ cell frequencies on days 3 and 7 p.i. (Figure $8 \mathrm{~A}$ ), and a reduced frequency of $\mathrm{CD}^{+} \mathrm{Foxp}^{+}$cells (Tregs; Figure $8 \mathrm{~B}$ ) on day 3 p.i. H1N1-infected WT and $\mathrm{Mmp}-\mathrm{9}^{-/-}$mice had similar frequencies of $\mathrm{CD}^{+} \mathrm{T}$ cells (Figure 8C), B cells (Supplemental Figure 4A), NK cells (Supplemental Figure 4B), NK T cells (Supplemental Figure 4C), and $\gamma \delta$ T cells (Supplemental Figure 4D) on day 7 p.i.

The frequency of $\mathrm{CD} 8^{+} \mathrm{T}$ cells expressing two inhibitory cell surface receptors ( $\mathrm{T}$ cell immunoglobulin and mucin domain-containing-3 [Tim3]) and programmed death protein-1 [PD1]) was quantified in enzymatic lung digests using immunostaining methods after gating on $\mathrm{CD}^{+} \mathrm{T}$ cells. Infected $\mathrm{Mmp}-9^{-/-}$mice had higher frequencies of $\mathrm{Tim}^{-} \mathrm{PD} 1^{-}$double-negative $\mathrm{CD}^{+} \mathrm{T}$ cells and lower frequencies of Tim3-expressing $\mathrm{CD}^{+} \mathrm{T}$ cells than infected WT mice (Figure 8D) on day 7 p.i. The frequencies of $\mathrm{PD} 1^{+}$and $\mathrm{Tim}^{+} \mathrm{PD} 1^{+}$ double-positive $\mathrm{CD}^{+} \mathrm{T}$ cells were similar in H1N1-infected WT and $\mathrm{Mmp}^{-9^{--}}$mice.

H1N1-infected Mmp-9-/- mice had higher frequencies of $\mathrm{CD}^{+}$lymphocytes and lower NK cell frequencies in BALF samples on day 7 p.i. than infected WT mice. Infected WT and $\mathrm{Mmp}^{-9^{-1}}$ mice had similar frequencies of $\mathrm{CD}^{+}$lymphocytes (Supplemental Figure 5, A-C). Intracellular staining for Tnf- $\alpha$, Ifn- $\gamma$, and Il-17 was performed on $\mathrm{CD}^{+}$and $\mathrm{CD} 8^{+} \mathrm{T}$ cells and NK cells in BAL leukocyte samples from WT and Mmp- $9^{-1-}$ mice on day 7 p.i. H1N1-infected Mmp- $9^{-/-}$mice had higher frequencies of Tnf- $\alpha^{+}$Ifn- $\gamma^{+}$ double-positive CD4 ${ }^{+} \mathrm{T}$ cells (Figure 9, A and B); Tnf- $\alpha^{+}$Ifn- $\gamma^{+}$double-positive and single-positive Ifn- $\gamma^{+}$ CD8 ${ }^{+}$lymphocytes (Figure 9, C and D); and Tnf- $\alpha^{+}$Ifn- $\gamma^{+}$double-positive NK cells (Figure 9, E and F). The expression of the inhibitory receptor, killer cell lectin-like receptor subfamily G-member-1 (Klrg1), was also quantified in $\mathrm{BAL} \mathrm{CD} 4^{+}$and $\mathrm{CD}^{+}$lymphocytes, as a marker of effector-cell activity. BAL CD8 ${ }^{+}$lymphocytes in H1N1-infected Mmp-9-/- mice had higher Klrg1 expression than CD8 ${ }^{+}$lymphocytes from IAV-infected WT mice (Figure 9G). There were no differences in K1rg1 expression on CD4 ${ }^{+}$cells in H1N1-infected WT and Mmp-9-/- mice (Figure $9 \mathrm{H}$ ).

Mmp-9-1- mice have reduced lung injury during H1N1 infection. As pulmonary inflammation can promote lung injury, we measured readouts of lung injury in H1N1-infected WT and $\mathrm{Mmp}^{-9^{--}}$mice. H1N1-infected 
A

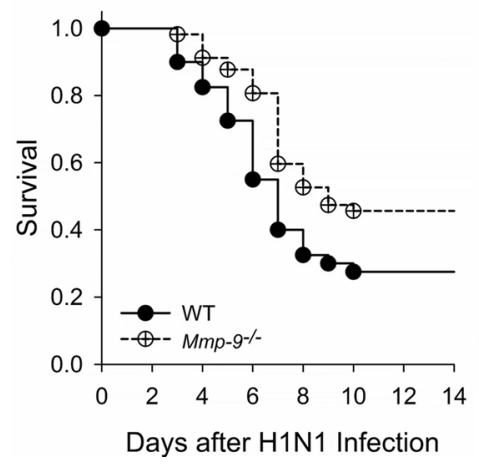

B

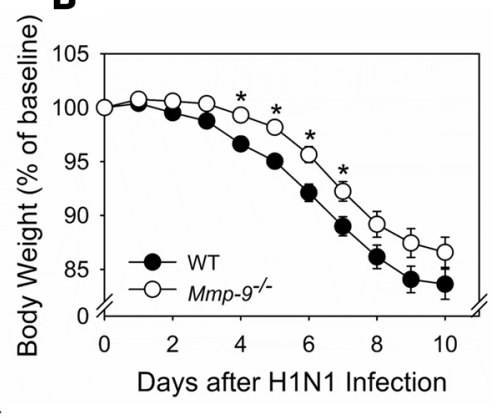

Figure 3. Mmp-9-/- mice were protected from H1N1-induced mortality and weight loss. WT $(n=40)$ and $M m p-9^{-/-}(n=57)$ mice were infected with an $\mathrm{LD}_{80}$ inoculum of $\mathrm{H} 1 \mathrm{~N} 1$ by the intranasal route. (A) Survival was monitored twice daily and Kaplan-Meier survival plots were generated. Gehan-Breslow-Wilcoxon analysis revealed significant differences between the survival curves $(P=0.004)$. (B) Changes in body weight as a percentage of baseline body weight were recorded daily for the first 10 days after infection. Data are expressed as mean \pm SEM. Data were analyzed using ANOVA for repetitive measures ( $P<0.001$ for WT and $\mathrm{Mmp}^{-\mathrm{g}^{-/-} \text {mice versus }}$ uninfected mice belonging to the same genotype), followed by pairwise testing with 2 -tailed Student's $t$ tests. ${ }^{*} P<0.05$ versus WT mice at the same time point.

Mmp-9-/- mice had reduced lung injury versus WT mice as assessed by lower BALF albumin levels on days 7-10 p.i. (Figure 10A), and lower airway/alveolar hemorrhage as assessed by lower BALF hemoglobin levels on days 7-10 p.i. (Figure 10B). WT mice developed perturbations in respiratory mechanics following $\mathrm{H} 1 \mathrm{~N} 1$ infection as assessed by increased tissue elastance and reduced quasi-static compliance (markers of lung stiffness) when compared with uninfected WT mice (Figure 10C and Supplemental Figure 6). However, $M m p-9^{-/-}$mice were substantially protected from H1N1-induced increases in lung elastance and reductions in quasi-static compliance, indicating that they were protected from H1N1-induced lung injury.

Mmp-9 mediates E-cadherin shedding in vitro $(19,23)$. The shedding of this adherens junctional protein from lung epithelial cells by Mmp-7 contributes to alveolar capillary barrier injury in murine lung injury models (24). Thus, we investigated whether infected $M m p^{-9^{-1}}$ mice have reduced shedding of E-cadherin to explain their reduced H1N1-induced lung injury. BALF soluble E-cadherin levels were lower at baseline and after 3-7 days p.i. in $M m p-9^{-/-}$mice versus WT mice (Figure 10D), indicating that shedding of E-cadherin is reduced in $\mathrm{Mmp}^{-9^{-/}}$mice.

Mmp-9 deficiency in lung parenchymal cells protects mice from H1N1-induced mortality. Mmp-9 is expressed by activated lung epithelial cells and leukocytes $(13,25)$. To identify the crucial cellular sources of Mmp- 9 during IAV infection in mice, we generated Mmp-9 BM chimeras and infected them with H1N1. WT mice transplanted with either Mmp-9-/- or WT BM had similar, low (20\%-40\%) survival rates following H1N1 infection (Figure 11A). Mmp- $9^{-/}$recipient mice transplanted with either WT or Mmp-9-/ $\mathrm{BM}$ had similar survival rates that were higher $(\sim 60 \%)$ than WT recipients of either Mmp- $-^{-/-}$or WT BM (Figure 11B). Thus, Mmp-9 deficiency in lung parenchymal cells protects mice from IAV-induced mortality.

H1N1 induces MMP-9 release by epithelial cells and increases IAV titers in vitro. To investigate whether IAV infection is sufficient to induce MMP-9 release by airway epithelial cells, we infected human bronchial epithelial cell (HBEC) cultures with H1N1 and measured the release of MMP-9 protein. H1N1 induced the release of MMP-9
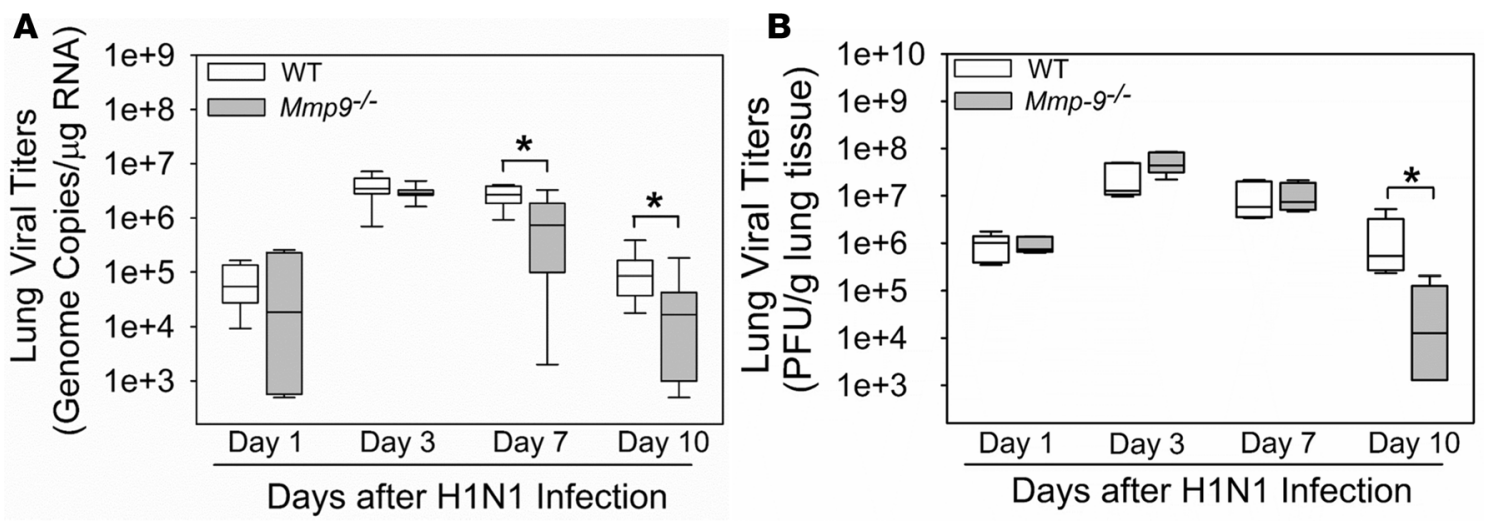

Figure 4. H1N1-infected Mmp-9-/- mice had lower lung IAV titers than H1N1-infected WT mice. WT and Mmp-9-/- mice (5-8 mice/group) were infected with an $\mathrm{LD}_{20}$ inoculum of $\mathrm{H} 1 \mathrm{~N} 1$ by the intranasal route. At postinfection intervals, viral titers in homogenates of lung samples were measured using qRT-PCR (A) or multifoci staining assays (B). The box-and-whisker plots show the medians and the 25th and 75 th percentiles, and the whiskers show the 10 th and 90 th percentiles. Data were analyzed with 1-way ANOVAs followed by pair-wise testing with Mann-Whitney $U$ tests. ${ }^{*} P<0.05$ versus the group indicated. 


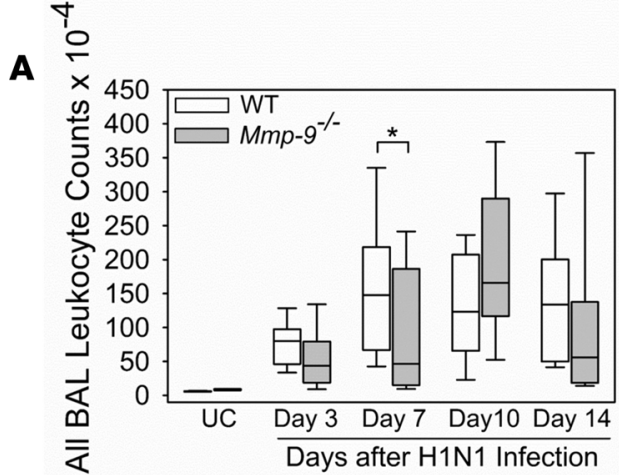

C

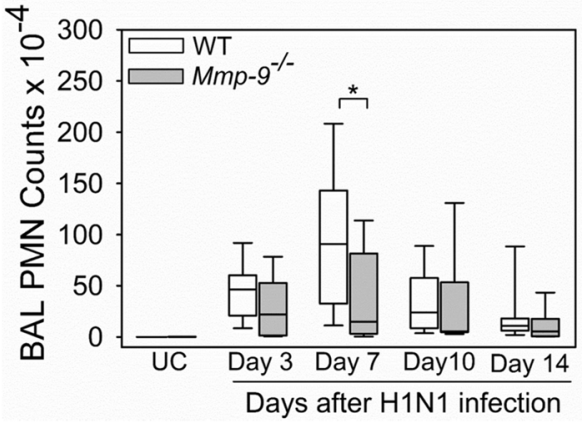

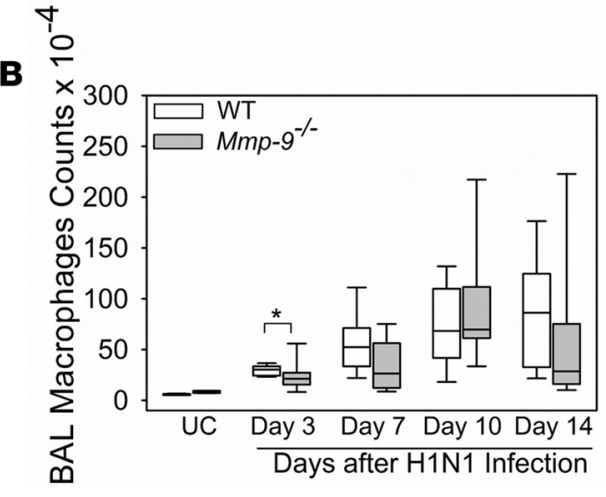

D

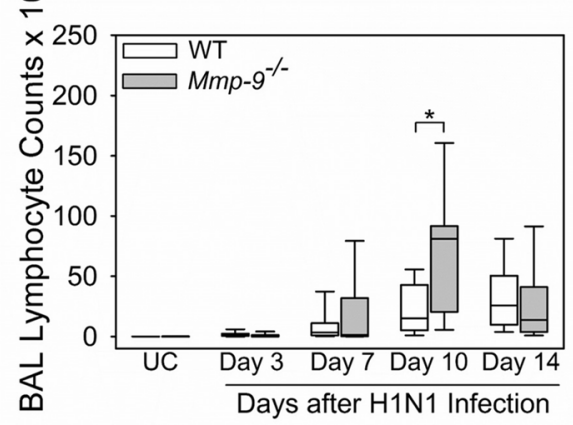

Figure 5. H1N1-infected Mmp-9-/- mice had lower early-phase BAL PMN and macrophage counts but higher latephase BAL lymphocyte counts than H1N1-infected WT mice. WT and Mmp-9-/- mice (8-12 mice/group) were infected with an $\mathrm{LD}_{20}$ inoculum of $\mathrm{H} 1 \mathrm{~N} 1$ by the intranasal route, or not infected as a control [UC, 9 mice]). At postinfection intervals, all leukocytes (A), PMNs (B), macrophages (C), and lymphocytes (D) were counted in bronchoalveolar lavage (BAL) samples. The box-and-whisker plots show the medians and the 25th and 75th percentiles, and the whiskers show the 10 th and 90 th percentiles. Data were analyzed with 1-way ANOVAs followed by pair-wise testing with Mann-Whitney $U$ tests. ${ }^{*} P<0.05$ versus the group indicated.

from HBECs by 48 hours p.i. (Figure 12A). Inhibiting extracellular MMP-9 activity by adding a neutralizing antibody to HBEC cultures reduced viral titers in culture supernatants (Figure 12B). Antibody-mediated MMP-9 inhibition also partially rescued the loss of transepithelial electrical resistance (TEER) occurring in H1N1-infected confluent HBEC monolayers, a measure of HBEC injury (Supplemental Figure 7).

MMP-9 does not cleave H1N1 hemagglutinin or neuraminidase. During IAV infection, some viral proteins require proteolytic activation to promote IAV infection of target cells (e.g., hemagglutinin [HA]). We assessed whether viral proteins (HA and neuraminidase [NA]) are substrates for MMP-9. The protease substrate specificity server (PROSPER) for cleavage predication analysis (26) predicted that human MMP-9 cleaves both HA subunit 1 (HA1) and NA subunit 1 (NA1) at multiple sites (Supplemental Table 1). However, active human MMP-9 had no activity against H1N1-derived HA1 (Supplemental Figure 8A), and minimal activity against H1N1-derived NA1 in vitro even when MMP-9 was tested at a high concentration (Supplemental Figure 8B).

\section{Discussion}

We report for the first time to our knowledge that circulating MMP-9 levels are increased in IAV-infected human subjects and H1N1 IAV-infected WT mice. Mmp-9 lung levels are also increased in IAV-infected WT mice, especially in airway epithelial cells and leukocytes. Mmp-9 deficiency in mice leads to less severe IAV disease than that observed in WT mice as assessed by lower mortality, reduced loss of body weight, and reduced IAV-induced lung injury. The improved survival in IAV-infected mice is likely due to their reduced lung injury and their lower lung viral burdens (Figure 13). The reduced lung injury in IAV-infected Mmp- $9^{-/-}$mice may be due, in part, to decreased E-cadherin shedding (with reduced impairment of epithelial barrier function), and reduced accumulation of myeloid leukocytes and their deleterious products in 
A

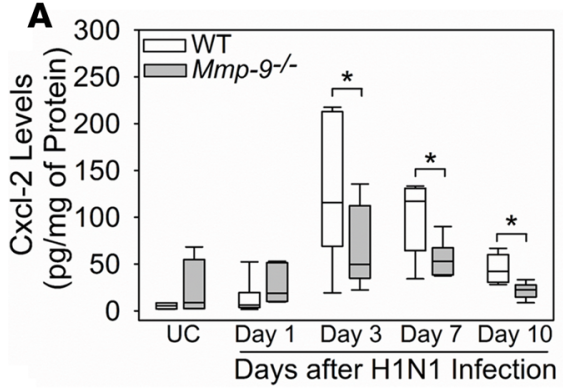

D

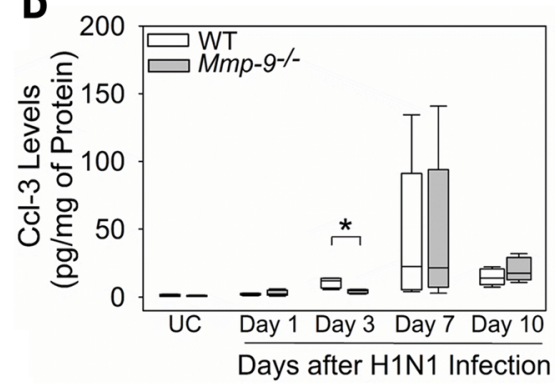

G

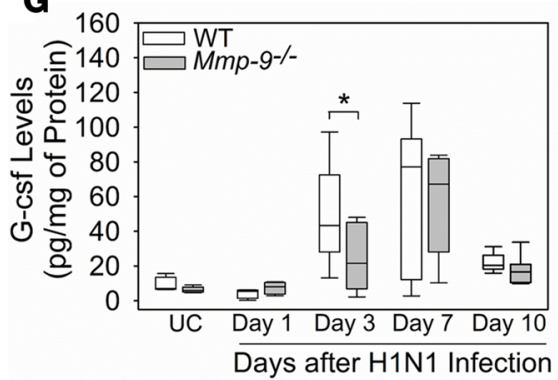

B

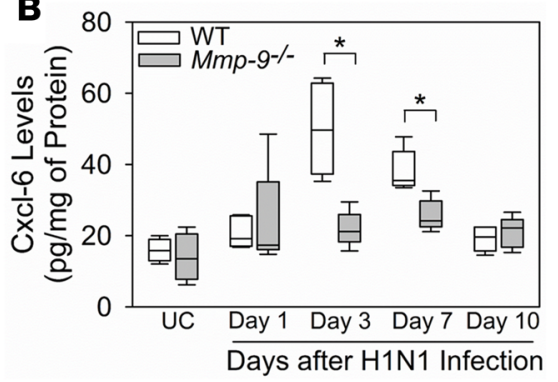

E

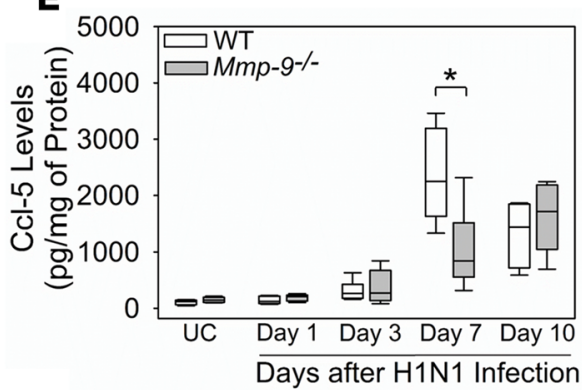

H

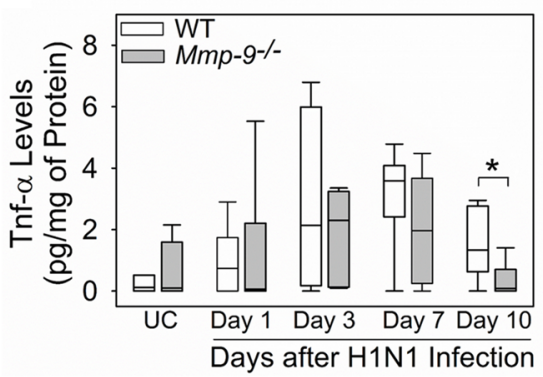

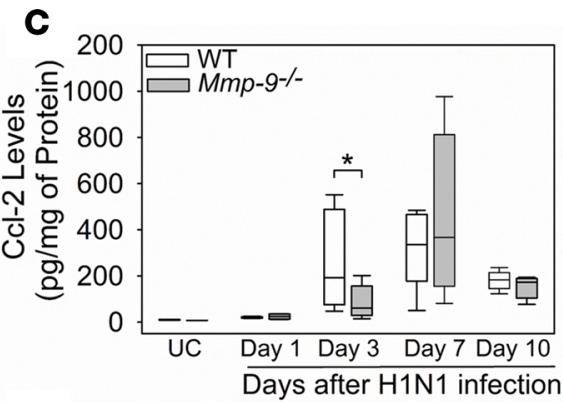

F

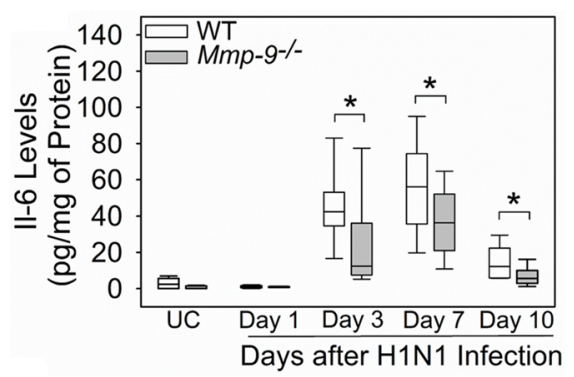

I

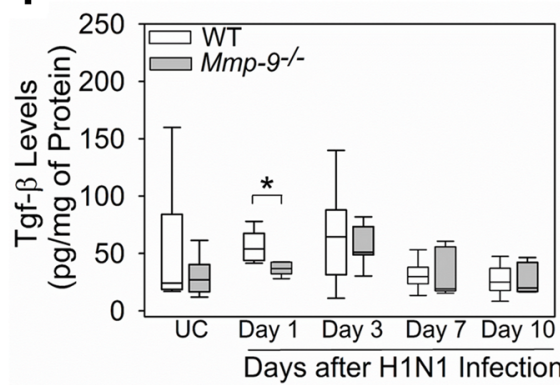

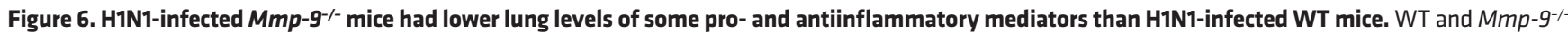
mice (4-10 mice/group) were infected with an LD $_{20}$ inoculum of H1N1 by the intranasal route. Uninfected control (UC) mice served as controls. At postinfection intervals, lungs were removed, and lung levels of Cxcl-2 (A), Cxcl-6 (B), Ccl-2 (C) Ccl-3 (D), Ccl-5 (E), II-6 (F), G-csf (G), Tnf- $\alpha$ (H), and Tgf- $\beta$ (I) were measured using ELISAs. Lung levels of mediators were normalized to lung total protein levels. The box-and-whisker plots show medians and 25 th and 75 th percentiles, and the whiskers showing 10 th and 90 th percentiles. Data were analyzed with 1 -way ANOVAs followed by pair-wise testing with Mann-Whitney $U$ tests. ${ }^{*} P<0.05$ versus the group indicated.

the lung (Figure 13). The reduced lung viral burdens in IAV-infected $M m p-9^{-1-}$ mice may be due to a more effective adaptive immune response to IAV developing in their lungs (Figure 13). Surprisingly, Mmp-9 is required for the generation of a robust type I IFN response to H1N1 IAV. Mmp-9 BM chimera experiments showed that Mmp-9 deficiency in lung parenchymal cells protects mice from IAV-induced mortality. Thus, our data show that MMP-9 is a key host protein that promotes IAV infection and could be targeted to develop novel therapies to limit IAV morbidity and mortality.

MMP-9 expression during IAV infection. MMP-9 plasma levels were more than 20 -fold higher in patients infected with H1N1 IAV and seasonal IAV than uninfected controls. Microvascular endothelial cells and circulating PMNs, monocytes, and lymphocytes are likely to be the main source of plasma MMP-9 during IAV infection, as these cells secrete MMP-9 when activated (27). Increased levels of various host proteinases including trypsin (which can activate pro-MMP-9), have been linked with the severity of the cytokine storm and end-organ injury that can develop in IAV-infected subjects $(28,29)$.

Mmp-9 protein levels in whole lung samples increased as early as day 3 p.i. in IAV-infected WT mice and remained elevated on day 10 p.i. Based on the results of our immunostaining studies, the early increase in lung Mmp-9 levels may be due to early PMN influx into the lungs and the release of preformed Mmp-9 protein by these cells $(9,12)$. The increased lung Mmp-9 protein levels on days 7-10 p.i. likely derive from Mmp-9 that is transcribed in airway epithelial cells, macrophages, CD4 ${ }^{+} \mathrm{T}$ cells, and B cells (12). During IAV infection, several transcription factors (including NF- $\mathrm{kB}$ and AP-1) are activated in the lung $(30,12)$. 
A

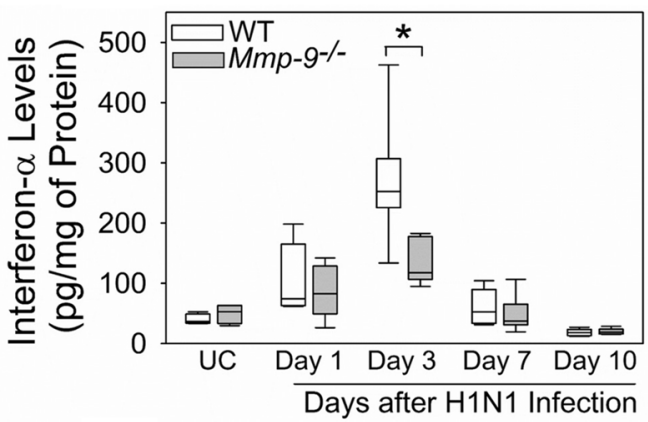

C

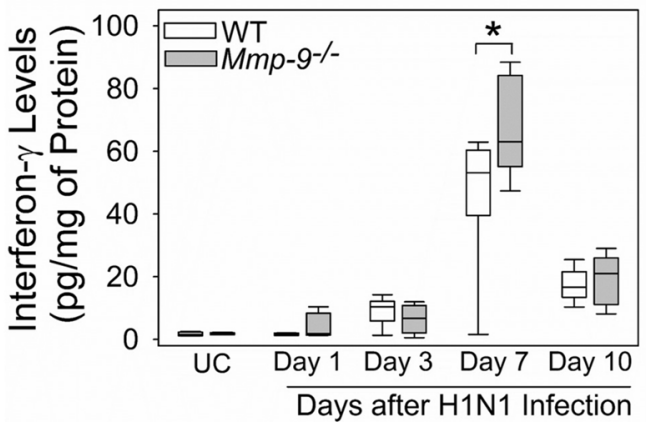

B

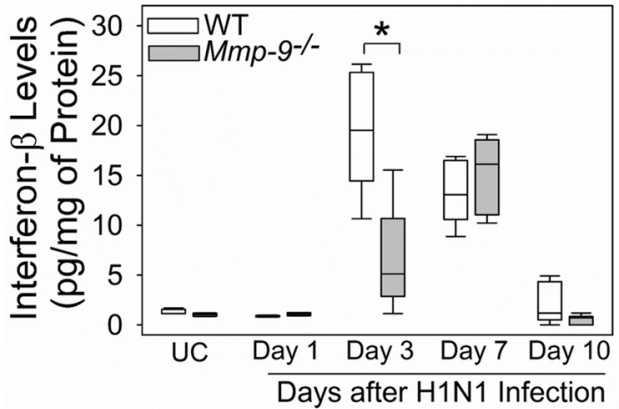

Figure 7. H1N1-infected Mmp-9-/- mice had reduced lung levels of type I interferons but increased lung levels of interferon- $\gamma$. WT and $\mathrm{Mmp9}^{-1-}$ mice (4-9 mice/group) were infected with an $\mathrm{LD}_{20}$ inoculum of $\mathrm{H} 1 \mathrm{~N} 1$ by the intranasal route. At postinfection intervals lung levels of interferon- $\alpha(\mathbf{A})$, interferon- $\beta$ (B), and interferon- $\gamma(\mathbf{C})$ were measured in lungs from infected mice or uninfected controls (UC; 8-9 mice/group) using ELISAs and levels of these mediators were normalized to lung total protein levels. The box-and-whisker plots show the medians and 25th and 75 th percentiles, and the whiskers show the 10th and 90th percentiles. Data were analyzed with 1-way ANOVAs followed by pair-wise testing with Mann-Whitney $U$ tests. ${ }^{*} P<0.05$ versus the group indicated.

Mmp-9 expression in lung macrophages is induced via the TLR7/NF- $\kappa B$ pathway in IAV-infected mice (31). Interestingly, Mmp-9 expression was increased in murine lungs and human small airway epithelial cells infected with respiratory syncytial virus (RSV) via the type I IFN pathway and RIG-I-like receptors (32). Thus, the increases in lung type I IFN levels detected on day 3 p.i. in IAV-infected WT mice may have contributed to the increased Mmp-9 expression observed in airway epithelium.

Mmp-9 deficiency in lung structural cells protected mice from IAV-induced mortality. Although activated endothelial cells and fibroblasts express MMP-9 (33), airway epithelial cells are likely to be the most important source of Mmp-9 in IAV-infected mice based on our lung immunostaining results, and

A $\stackrel{0}{\overline{0}}$

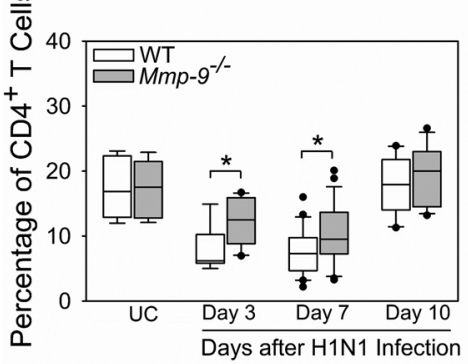

C

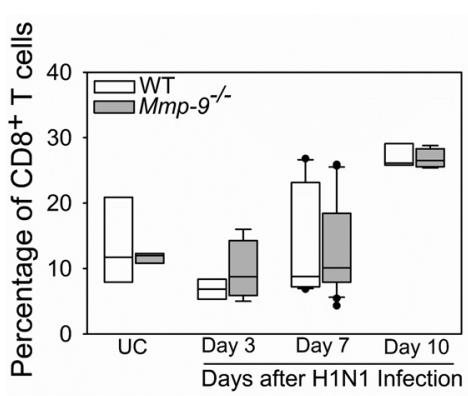

B

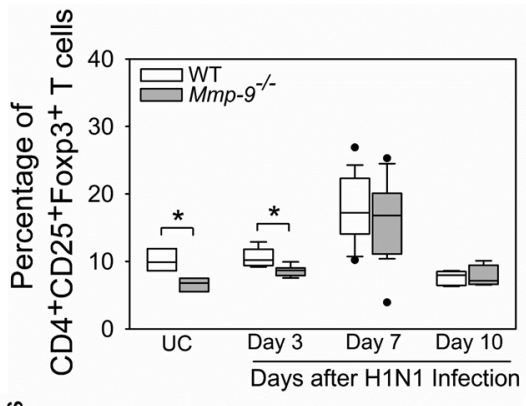

D $\frac{\infty}{\bar{d}}$

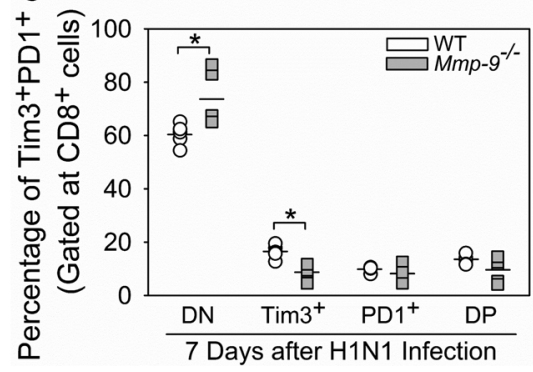

Figure 8. H1N1-infected Mmp-9-/- mice had higher frequencies of lung $\mathrm{CD4}^{+} \mathrm{T}$ cells and lower frequencies of lung $\mathrm{CD}_{25}{ }^{+} \mathrm{Foxp}^{+} \mathrm{CD4}^{+} \mathrm{T}$ cells, and reduced expression of 2 inhibitory receptors (Tim3 and PD1) on CD8 ${ }^{+}$ T cells compared with H1N1-infected WT mice. WT and $\mathrm{Mmp}-\mathrm{9}^{-/-}$mice were infected with an $\mathrm{LD}_{20}$ inoculum of $\mathrm{H} 1 \mathrm{~N} 1$ via the intranasal route, and the lungs were removed on day 3 (3-11 mice per group), day 7 (17-29 mice per group), and day 10 (3-11 mice per group) postinfection (p.i.). Uninfected (UC) mice (3-6 mice per group) served as controls. Cells in enzymatic lung digests were immunostained for $\mathrm{CD}_{4} 5^{+} \mathrm{CD} 4^{+}$and $\mathrm{CD} 45^{+} \mathrm{CD} 8^{+} \mathrm{T}$ cells, $\mathrm{CD} 4{ }^{+} \mathrm{CD} 25^{+}$ Foxp $3^{+} \mathrm{T}$ regulatory cells, and inhibitory surface receptors (Tim3 and PD1), and analyzed using flow cytometry. The percentages of lung $C D 45^{+}$leukocytes that were $C D 4^{+} T$ cells (A) and $C D 8^{+} T$ cells (C) were quantified. Gating was conducted on the $\mathrm{CD} 4^{+}$and $\mathrm{CD} 8^{+} T$ cells for lymphocyte subsets including $\mathrm{CD}^{+} \mathrm{CD} 25^{+} \mathrm{Foxp}^{+} \mathrm{T}$ regulatory cells (B) and $C D 8^{+} T$ cells that were Tim3 and PD1 double negative, $\mathrm{Tim3}^{+}, \mathrm{PD}^{+}$, or $\mathrm{Tim} 3$ and $\mathrm{PD1}$ double positive on day $7 \mathrm{p}$.i. (D). The box-and-whisker plots show the medians and 25th and 75th percentiles, and the whiskers show the 10th and 90th percentiles. Data were analyzed with 1-way ANOVAs followed by pair-wise testing with Mann-Whitney $U$ tests. ${ }^{*} P<0.05$ versus the group indicated. 
A
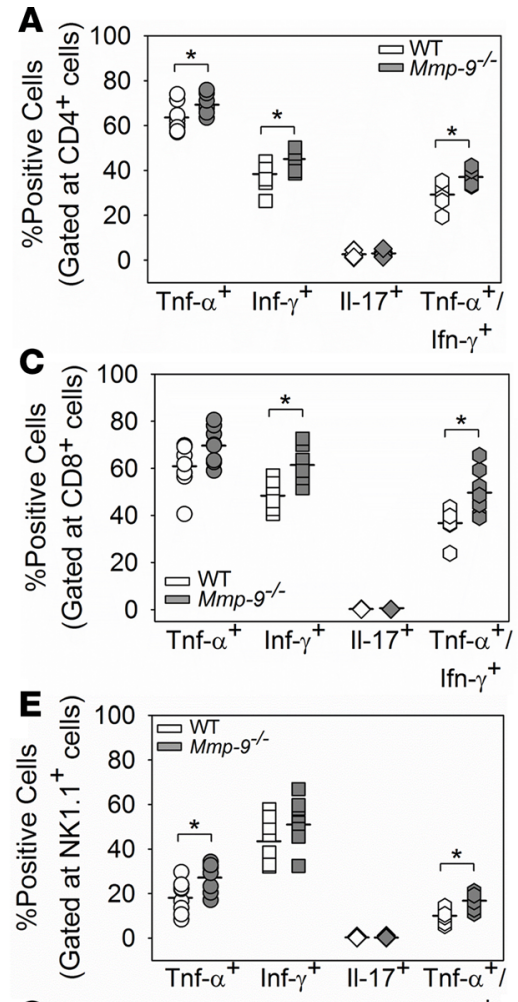

G

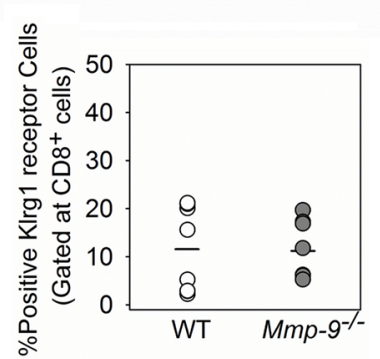

B

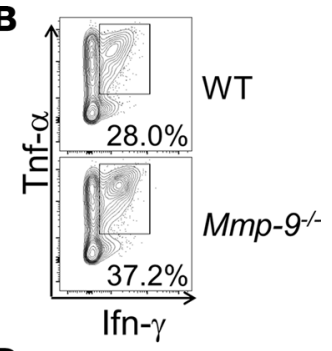

D

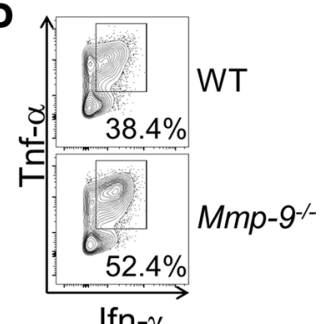

$\mathbf{F}$
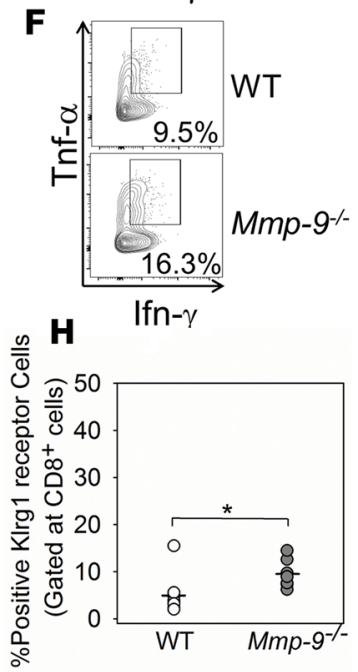

Figure 9. Mmp-9-/- mice had increased numbers of BAL CD4 ${ }^{+}$lymphocytes, CD8+ Iymphocytes, and NK cells that were Tnf- $\alpha^{+}$Ifn- $\gamma^{+}$double positive than WT mice following H1N1 infection. WT and Mmp-9-1mice were infected with an $\mathrm{LD}_{20}$ inoculum of $\mathrm{H} 1 \mathrm{~N} 1$ via the intranasal route (8 mice per group), and BAL was performed on day 7 p.i. BAL leukocytes were immunostained to identify $\mathrm{CD}^{+}$and $\mathrm{CD} 8^{+} \mathrm{T}$ cells, NK cells (NK1.1+), and to measure intracellular cytokines (Tnf- $\alpha$, Ifn- $\gamma$, and II-17) in these cells using flow cytometry. Frequencies of BAL CD4 ${ }^{+} T$ cells ( $\mathbf{A}$ and $\mathbf{B}), \mathrm{CD}^{+}$T cells ( $\mathbf{C}$ and $\mathbf{D}$ ), and NK cells (E and $\mathbf{F}$ ) are shown. Gating was conducted on Tnf- $\alpha^{+}$single-positive cells, Ifn- $\gamma^{+}$single-positive cells, $\mathrm{Il}-17^{+}$single-positive cells, and Tnf- $\alpha^{+} \mathrm{Ifn}-\gamma^{+}$double-positive cells. The horizontal bars show the mean values. The rectangles in the histograms (B, D, and F) show the Tnf- $\alpha^{+} \mid \mathrm{fn}-\gamma^{+}$double-positive cells in each T cell subset. ( $\mathbf{G}$ and $\mathbf{H}$ ) BAL leukocytes were immunostained and for the inhibitory receptor, KIrg1, and markers of $\mathrm{CD}^{+}(\mathbf{C})$ and $\mathrm{CD} 8^{+} \mathrm{T}$ cells $(\mathbf{H})$ and analyzed using flow cytometry. Data were analyzed with 1-way ANOVAs followed by 2-sided Student's $t$ tests (A, C, and $\mathbf{E}$ ) or followed by 2 -sided Mann-Whitney $U$ tests (G and $\mathbf{H}$ ). ${ }^{*} P<0.05$ versus the group indicated.

studies showing that antibody-mediated neutralization of MMP-9 secreted by H1N1-infected human lung epithelial cultures leads to reduced viral titers in these cultures.

Mechanisms by which Mmp-9 deficiency in mice leads to better outcomes following IAV infection. The improved survival rates of IAV-infected $M m p-9^{-1-}$ versus WT mice is likely due to a combination of factors including their (a) lower lung viral burdens, (b) enhanced pulmonary adaptive immune response, and (c) less severe lung injury, as more severe lung injury is linked to increased mortality in other lung inflammation models $(34,35)$.

IAV lung burdens. Lung viral titers were similar in IAV-infected WT and $\mathrm{Mmp}^{-9^{--}}$mice up to day 3 p.i., indicating that Mmp-9 is not essential for IAV to enter and replicate within epithelial cells. From day 7 p.i. onwards, IAV-infected $M m p-9^{-/-}$mice had lower lung viral burdens than WT mice, which could be due to Mmp-9 deficiency (a) increasing antiviral (IFN) responses, (b) increasing pulmonary immune responses to IAV, (c) increasing viral clearance after initial infection in epithelial cells, and/or (d) reducing virion release from infected cells and impairing viral propagation.

We initially hypothesized that $M m p 9^{-1-}$ mice have greater antiviral cytokine (IFN) responses during IAV infections. Immune cells (including macrophages and dendritic cells [DCs]) and nonimmune cells produce type I IFNs (Ifn- $\alpha$ and Ifn- $\beta$ ) after sensing viral components via pattern-recognition receptors (36). Plasmacytoid DCs produce large quantities of Ifn- $\alpha$, and fibroblasts and epithelial cells mainly produce Ifn- $\beta$, and Ifn- $\gamma$ is secreted by T helper cells, cytotoxic T cells, NK cells, and macrophages (37). Activation of ubiquitously expressed type I IFN receptors triggers STAT activation and the transcription of IFN-stimulated genes in infected cells and nearby uninfected cells, leading to the secretion of proteins having antiviral effects $(36,37)$. Type I IFNs also promote antigen presentation and NK cell functions and stimulate the 

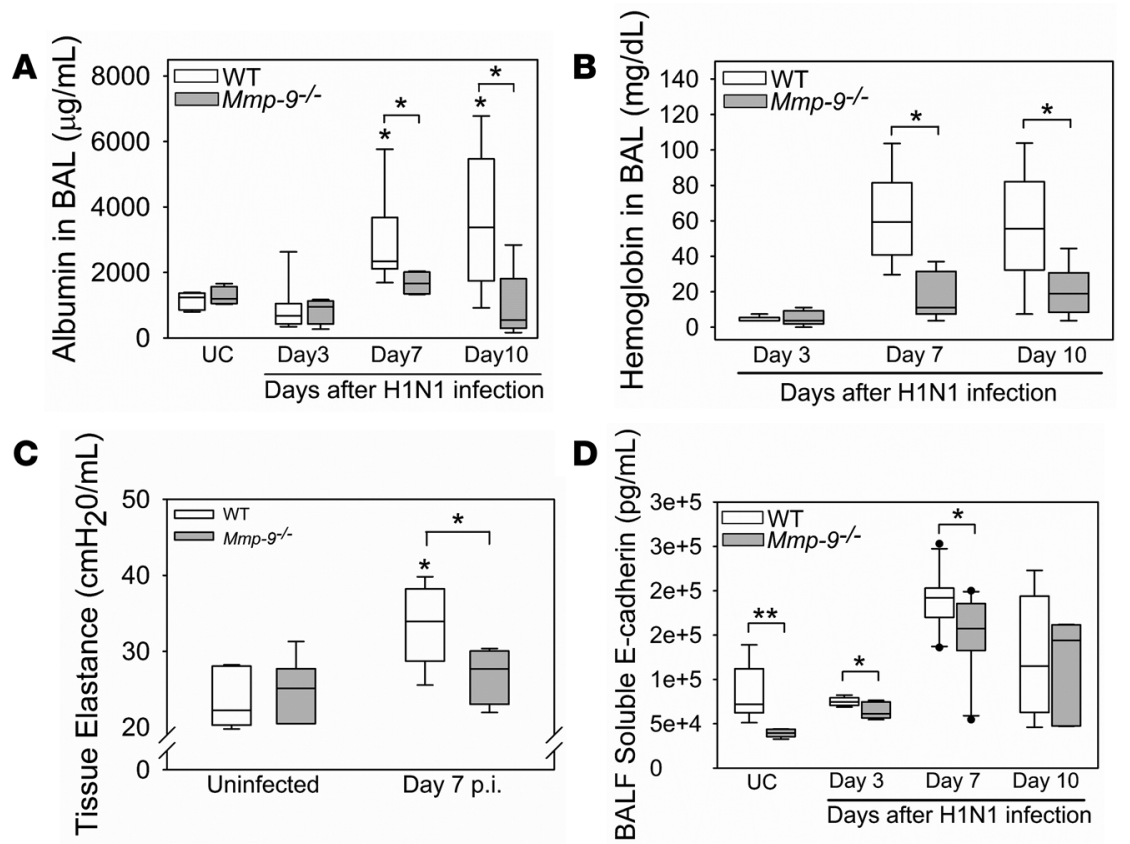

Figure 10. Mmp-9-/- mice were protected from lung injury following IAV infection. WT and $M m p-9^{-/-}$mice were infected with an $\mathrm{LD}_{20}$ inoculum of $\mathrm{H} 1 \mathrm{~N} 1$ by the intranasal route. At postinfection intervals, albumin $(\mathbf{A})$ and hemoglobin (B) levels were measured in BALF samples from H1N1-infected mice (4-11 mice/group) and in uninfected control (UC) mice (4-5 mice/group). ${ }^{*} P<0.05$ versus the group indicated or uninfected controls belonging to the same genotype. (C) Tissue elastance was measured in H1N1-infected mice on day 7 p.i. (8 mice per group) and uninfected control mice (8-9 mice per group) using a FlexiVent device. ${ }^{*} P<0.05$ versus the group indicated or UC mice belonging to the same genotype. (D) Soluble E-cadherin levels were measured in BALF samples from infected mice (4-11 mice per group) and uninfected control mice (6 mice per group) using an ELISA. ${ }^{*} P<0.05$; ${ }^{* *} P<0.01$ versus the group indicated. All box-and-whisker plots show the medians and 25th and 75th percentiles, and the whiskers show the 10th and 90th percentiles. All data were analyzed with 1-way ANOVA followed by pair-wise testing with Mann-Whitney $U$ tests.

development of high-affinity antigen-specific $\mathrm{T}$ and $\mathrm{B}$ cell responses $(36,37)$. Contrary to our hypothesis, we showed for the first time to our knowledge that Mmp-9 is required for the generation of a robust type I IFN response to IAV (Figure 7). The reduced type I IFN response in IAV-infected $M m p-9^{-1-}$ mice may contribute to their reduced lung injury and better survival compared with WT mice, as excessive type I IFN signaling (38) in patients with autoimmune diseases promotes tissue injury by (a) increasing host cell expression of autoantigens, (b) increasing immunostimulatory antigen-presenting cells thereby reducing immune tolerance and increasing autoreactive $\mathrm{T}$ cell generation, and (c) promoting $\mathrm{T}$ cell survival.

Ifn- $\gamma$ is secreted by $\mathrm{T}$ helper cells, cytotoxic $\mathrm{T}$ cells, NK cells, and macrophages (37). Ifn- $\gamma$ promotes adaptive immunity by recruiting $\mathrm{CD} 8^{+}$lymphocytes (39), inducing $\mathrm{CD} 4^{+} \mathrm{Th} 1$ differentiation (40), activating NK cells (41), and orchestrating T cell responses during IAV infection (42). Infected $M m p-9^{-/-}$mice had higher lung Ifn- $\gamma$ levels that peaked on day 7 p.i., coinciding with elevated Th1 $\mathrm{CD}^{+}$, cytotoxic $\mathrm{CD} 8^{+} \mathrm{T}$ cells, and Th1-like cytotoxic NK cell counts in their lungs. Thus, Mmp-9 deficiency in mice may lower lung viral burdens by increasing lung Ifn- $\gamma$ levels, thereby promoting a more effective $\mathrm{T}$ cell response to IAV.

The mechanisms by which Mmp-9 deficiency in mice alters the expression of IFNs in the lungs is unclear. During coxsackievirus type B3 infection, MMP-12 secreted by macrophages is internalized by virally infected cells, translocates to the nucleus, binds to the NFKBIA promoter, and drives transcription of I $\mathrm{KB} \alpha$, which promotes export of Ifn- $\alpha$ from the cells (43). Thus, one intriguing possibility is that Mmp-9 may serve as a transcription (co)factor that increases the expression type I IFNs and/or reduces the expression of IFN- $\gamma$. Although MMP-9 has not been reported to have intracellular activities, our future studies will investigate whether MMP-9 serves as a transcription (co)factor during IAV infection in mice.

Host inflammatory response. Viral clearance depends on Th1 humoral and cell-dependent responses. Release of mediators by IAV-infected airway epithelial cells increases leukocyte recruitment into the lung, and $\mathrm{CD} 8^{+} \mathrm{T}$ cell- and NK cell-mediated killing of infected cells, which is followed by tissue repair after viral clearance (27). However, IAV interaction with respiratory epithelium can initiate and amplify 
A

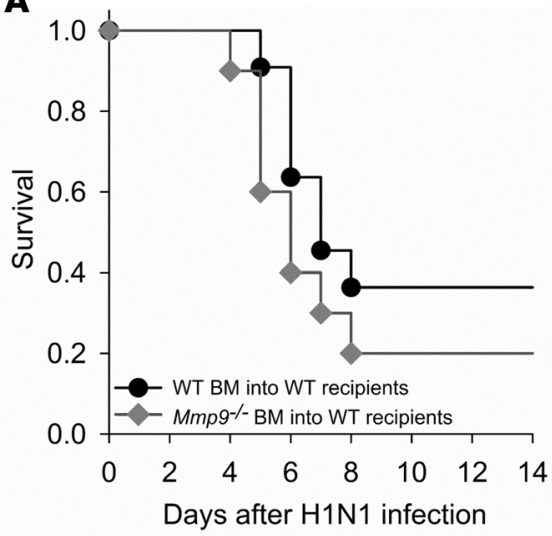

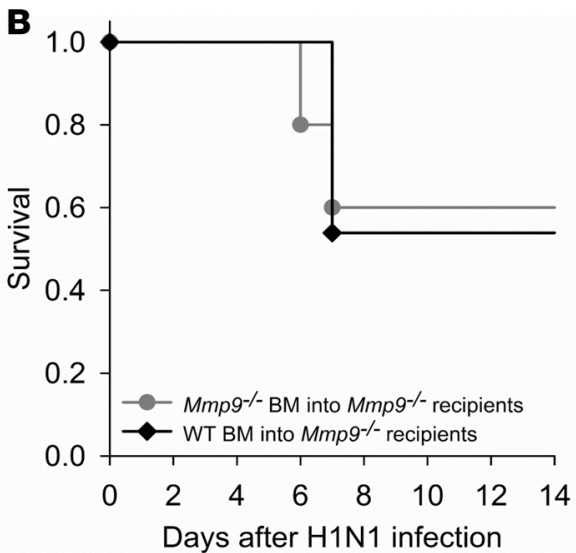

Figure 11. Mmp-9 deficiency in lung parenchymal cells led to reduced mortality following H1N1 infection. Bone mar-

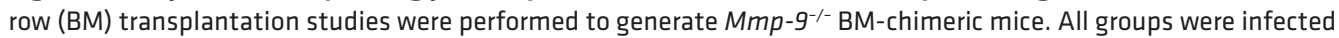
with an $\mathrm{LD}_{80} \mathrm{H} 1 \mathrm{~N} 1$ inoculum via the intranasal route and survival or moribundity was monitored daily for 14 days. (A) Kaplan-Meier survival curves for WT recipients with WT BM $(n=11)$ versus WT recipients with Mmp-9-/- BM $(n=10)$.

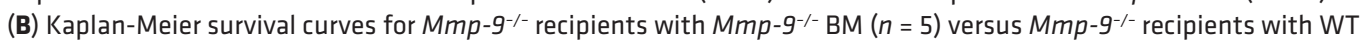
BM $(n=13)$. A Gehan-Breslow-Wilcoxon analysis was performed on the groups having the same recipient genotype. $P$ $=0.671(\mathbf{A})$ and $P=0.907(B)$.

IAV-associated cytokine storm (44), an excessively exuberant host response that causes severe tissue injury and increases mortality rates. During IAV infection, $M m p-9^{-/-}$mice had lower lung PMN and macrophage counts than WT mice, which may have contributed to their reduced lung injury and better survival, as these cells release proteinases, oxidants, and cytokines (permogens) that injure the alveolar capillary barrier (45). We report for the first time to our knowledge that IAV-infected Mmp-9-1 mice had an enhanced pulmonary adaptive immune response to IAV, with higher $\mathrm{CD} 4^{+} \mathrm{T}$ lymphocytes, Th1 $\mathrm{CD} 4^{+}$lymphocytes, Th1-like $\mathrm{CD} 8^{+}$lymphocytes, and NK cell counts, and lower frequencies of antiinflammatory Tregs. IAV-infected $M m p-9^{---}$mice had higher numbers of $\mathrm{CD} 8^{+} \mathrm{T}$ cells lacking inhibitory (Tim3) receptors, indicating that these cells have increased effector activity.

IAV-infected $\mathrm{Mmp}-9^{-1-}$ mice also had higher frequencies of $\mathrm{CD} 8^{+} \mathrm{T}$ cells expressing the inhibitory receptor, Klrg1, in their lungs. Klrg1 has an ITAM inhibitory motif and is expressed by antigen-experienced $\mathrm{NK}$ and $\mathrm{CD}^{+}$and $\mathrm{CD} 8^{+} \mathrm{T}$ cells (46). The binding of the main ligand for Klrg1, soluble E-cadherin (sE-cadherin), to this receptor induces lymphocyte dysfunction including reduced cytolytic activity of NK cells (by inhibiting degranulation and IFN- $\gamma$ release), reduced $\mathrm{CD} 8^{+} \mathrm{T}$ cell proliferation and differentiation towards long-term memory cells (47), and increased CD8 $8^{+} \mathrm{T}$ cell senescence (48). In HIV-infected patients, higher sE-cadherin levels are associated with a higher viral load, defective HIV-specific CD8 ${ }^{+}$lymphocytes, and disease progression (49). In our study, the lower sE-cadherin lung levels detected in IAV-infected $M m p^{-9^{-/}}$mice may have reduced the senescence of Klrg1-expressing Tnf- $\alpha^{+} \mathrm{Ifn}-\gamma^{+}$and Ifn- $\gamma^{+} \mathrm{CD} 4^{+}$and $\mathrm{CD} 8^{+} \mathrm{T}$ cells, instead promoting their efficient proliferation and cytotoxic activity, thereby increasing viral clearance (Figure 13).

MMP-9 proteolytically activates chemokines in vitro to regulate their biologic activity (50), and regulates lung inflammatory responses in other models $(16,17)$. We found no evidence that Mmp-9 proteolytically degrades mediators in IAV-infected mice, as lung levels of most mediators measured (Cxcl-2, Ccl-2, Ccl-3, Ccl-5, Il-6, G-csf, and Tnf- $\alpha$ ) were lower (rather than higher) in IAV-infected $M m p-9^{-1-}$ versus WT mice. The lower lung levels of these mediators in $\mathrm{Mmp}-9^{-/-}$mice may be due to the reduced accumulation of PMNs and macrophages in their lungs, as these cells are key sources of these mediators. The lower lung levels of these mediators in infected $\mathrm{Mmp}^{-9^{-/}}$mice may have contributed to their better outcomes, as some of these mediators promote injury to the alveolar capillary barrier (Tnf- $\alpha$ and Ccl-3), and amplify systemic and lung inflammatory responses to infection (Tnf- $\alpha$ and Ccl-3) $(34,51)$. Cxcl-2 and G-csf promote PMN degranulation and prolong PMN survival, and thus the lower lung levels of Cxcl-2 and G-csf may have reduced lung PMN counts and PMN-mediated lung injury in IAV-infected $M m p-9^{-/-}$versus WT mice.

Lung injury. The less severe lung injury detected in IAV-infected $\mathrm{Mmp-9^{-/- }}$ mice could be due to their lower lung PMN and macrophage counts, as these cells release proteinase, oxidants, and permogens that can injure 
A

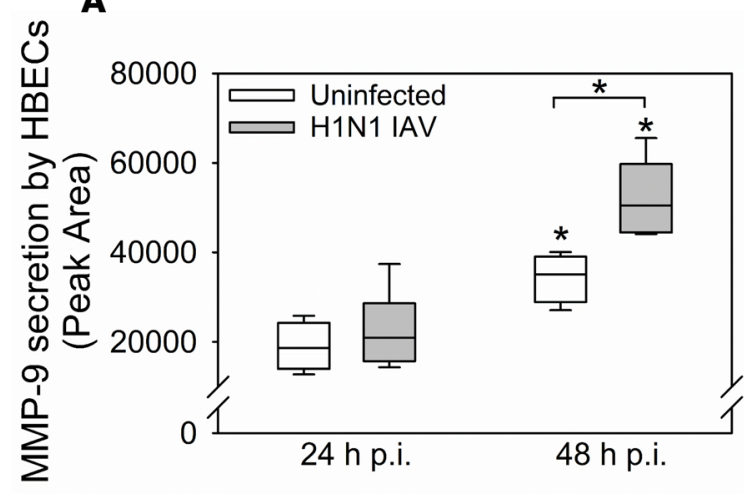

B

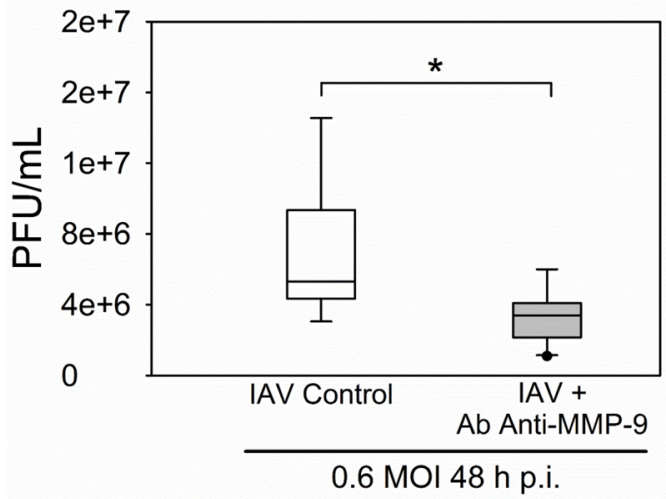

Figure 12. H1N1 increased MMP-9 release by epithelial cells and secreted MMP-9 promoted higher epithelial H1N1 titers in vitro. (A) Human bronchial epithelial cells (HBECs) were infected with H1N1 at a multiplicity of infection of 3 for up to 48 hours. MMP-9 levels were measured in culture supernatants using gelatin zymography and band intensities were quantified. ${ }^{*} P<0.05$ versus the group indicated or the same experimental condition at 24 hours $p . i$. (B) HBECs were infected with at a multiplicity of infection of 0.6 in the presence or absence of a neutralizing antibody against MMP-9. Viral titers were measured after 48 hours ( $n=3$ separate experiments). ${ }^{*} P<0.05$. All box-and-whisker plots show the medians and 25 th and 75 th percentiles, and the whiskers show the 10th and 90th percentiles. All data were analyzed with 1-way ANOVAs followed by pair-wise testing with Mann-Whitney $U$ tests.

the alveolar capillary barrier $(45,52,53)$. It is noteworthy that lung levels of Tnf- $\alpha$ (a permogen) were lower in infected $\mathrm{Mmp}-9^{-/-}$mice. IAV-infected $\mathrm{Mmp}^{-9^{-/}}$mice also had reduced E-cadherin shedding in their lungs as assessed by lower lung sE-cadherin levels. E-cadherin is a key component of adherens junctions, which contribute to the integrity of the lung epithelial barrier (54). Shedding of E-cadherin leads to increased epithelial permeability and increased lung injury. A prior study reported that adding active MMP-9 to the apical aspect of HBEC monolayers in vitro resulted in impaired barrier function associated with reduced staining for junctional proteins (including E-cadherin), increased access of virus to the basolateral epithelial surface, and increased infection efficiency (18). This activity was hypothesized to be due to MMP-9 cleaving junctional proteins leading to epithelial cell injury, but shedding of junctional proteins by MMP-9 was not evaluated (18). Another study reported that MMP-9 cleaves E-cadherin directly from ovarian carcinoma epithelial cell lines in vitro (19). Whether Mmp-9 mediates E-cadherin shedding in vivo with functional consequences has not been assessed previously. Our study is the first to our knowledge to report that shedding of E-cadherin is reduced in the lungs of $\mathrm{Mmp}-9^{-/-}$mice and associated with protection from loss of barrier function in the lung. Mmp-9 may directly cleave E-cadherin from lung epithelial cells during IAV infection, as even uninfected Mmp- $9^{-1-}$ mice had lower BALF sE-cadherin levels than uninfected WT mice, and antibody-mediated neutralization of MMP-9 secreted by HBECs reduced HBEC injury (loss of TEER) in vitro. However, it is also possible that during IAV infection, $M m p 9^{-1-}$ mice have increased expression or secretion of other proteinases known to mediate E-cadherin shedding $(23,55,56)$.

Cleavage of viral proteins. We considered the possibility that MMP-9 could hinder clearance of the virus or virions released from infected cells by cleaving viral proteins. Initially, IAV attaches to sialic acid-containing glycan receptors on the host cell surface the via its HA (57). Cleavage of HA1 is required for IAV to bind to sialic acid groups and is mediated by trypsin (58) and other host proteinases (59). IAV is then internalized by endocytosis, and the viral genome is released into the cytoplasm through HA-mediated membrane fusion and virus uncoating. The viral RNA is transported into the nucleus, where new viral components are transcribed to form progeny virions that bud from the apical cell membrane with HA and NA on their surface (60). NA cleaves sialic acid from glycans on host cells and the emerging virions, thereby releasing progeny viruses from infected cells and preventing virus aggregation by preventing HA-HA interactions. NA cleaves sialic acids from respiratory tract mucins to facilitate IAV entry into airway epithelial cells. We found no evidence that MMP-9 cleaves HA1, and MMP-9 cleaved NA only very weakly when tested at higher concentrations in vitro. Thus, it is unlikely that $M m p-9$ deficiency in mice improves outcomes via loss of Mmp-9-mediated cleavage of viral proteins.

Comparison with other studies. MMPs have not been well studied in IAV infection models. Membrane-bound MT1-MMP is the main interstitial collagen-degrading proteinase during IAV infections in mice (61). 


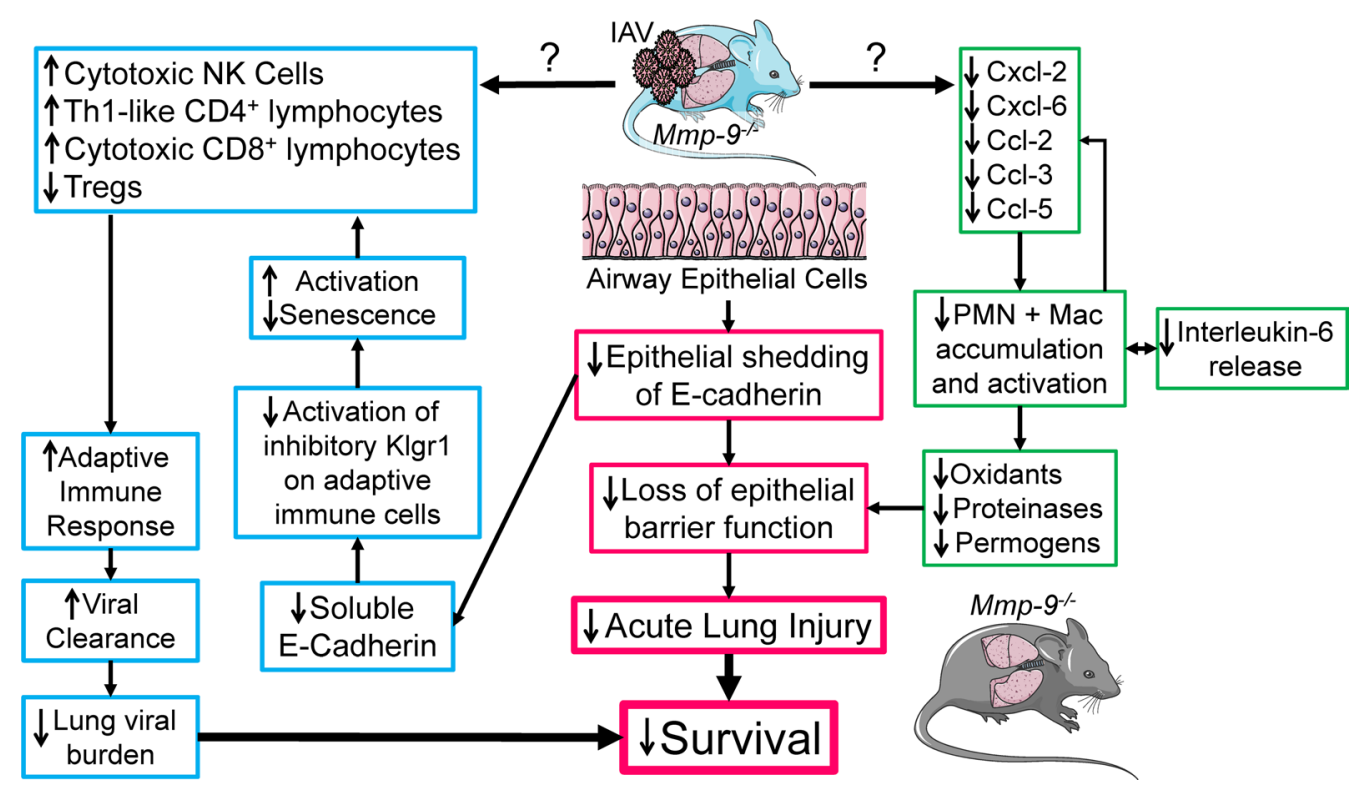

Figure 13. Mmp-9 deficiency in mice leads to increased survival following H1N1 IAV infection. H1N1-infected

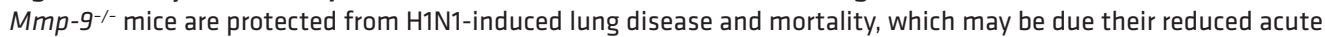
lung injury and their more effective adaptive immune response to H1N1 IAV when compared with H1N1-infected WT mice. The reduced lung injury in H1N1-infected $\mathrm{Mmp}^{-9^{-/-}}$mice may be due to reduced lung levels of cytokines and chemokines leading to reduced accumulation of PMNs and macrophages (Mac) in the lung. This, in turn, may reduce the lung burden of deleterious products released by these myeloid leukocytes including oxidants, proteinases, and permogens (such as Tnf- $\alpha$ ) that can promote lung injury. Reduced production of Cxc and Ccl chemokines and cytokines by the reduced numbers of myeloid leukocytes may contribute to the blunted recruitment of these cells into the lungs of H1N1-infected $\mathrm{Mmp}^{-\mathrm{g}^{-/-}}$mice. H1N1-infected $\mathrm{Mmp}^{-\mathrm{S}^{-/-}}$mice also have reduced shedding of the adherens junctional protein, E-cadherin, from lung epithelial cells, and reduced loss of epithelial barrier integrity compared with H1N1-infected WT mice. H1N1-infected $\mathrm{Mmp}^{-\mathrm{S}^{-/-}}$mice have an enhanced adaptive immune response characterized by increased numbers of effector cells including cytotoxic NK cells and CD8+ T lymphocytes, and Th1-like CD4 ${ }^{+}$ lymphocytes, along with reduced numbers of antiinflammatory T regulatory cells in their lungs when compared with H1N1-infected WT mice. Soluble E-cadherin is a ligand for the inhibitory receptor, KIrg1, on cytotoxic NK cells and CD8+ lymphocytes, and Th1-like CD4+ Iymphocytes. Thus, the lower lung levels of soluble E-cadherin in H1N1-infected Mmp-9/- mice may also reduce KIrg1 signaling in these adaptive immune cells, and thereby promote their activation and proliferation, and reduce their senescence. Together, these changes likely enhance the adaptive immune system to IAV leading to faster viral clearance, lower lung viral burdens, and improved survival.

Bradley et al. (62) reported that Mmp-9-- mice had higher lung viral loads and higher lung PMN counts following infection with the PR8 strain of IAV. Other studies report that PMN depletion is associated with increased morbidity and mortality (63) and delayed $\mathrm{T}$ cell response (64). Herein, we studied a different

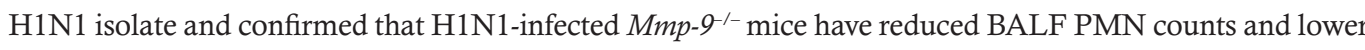
lung viral burdens. The reason for the differences in our results versus those of Bradley et al. is not clear. However, in contrast to the study of Bradley et al. (62), we conducted detailed time course experiments to quantify PMN counts and viral titers using much higher sample sizes for both readouts, and we used two different methods to quantify lung viral titers.

Limitations. We have not identified the underlying mechanisms by which Mmp-9 deficiency alters pulmonary immune responses and IFN levels in the lungs of IAV-infected mice. B cell subsets were not compared in the lungs of IAV-infected WT and $\mathrm{Mmp}-9^{-/-}$mice. Activated B cells and plasma cells produce antibodies that promote clearance of IAV, but can contribute to lung inflammation and injury. Regulatory B cells have antiinflammatory activities in other models (65). Studies of plasma levels and different forms of MMP-9 in larger cohorts of IAV-infected human subjects are needed to assess whether plasma MMP-9 levels can serve as a predictive or prognostic biomarker of severe disease or adverse outcomes in IAV-infected human subjects. It is not clear whether Mmp-9 cleaves mediators in the lungs of IAV-infected mice, as the commercially available ELISAs that we used to quantify lung levels of mediators may or may not detect cleavage products of mediators that are known to be substrates for MMP-9 (e.g., Cxcl-2, Cxcl-6, and Ccl-2; see ref. 66). 
Conclusions. Our results show that MMP-9 blood and lung levels are increased during IAV infections. Mmp-9 deficiency in mice leads to better outcomes following IAV infection by (a) increasing clearance of IAV from the lung likely by inducing a more effective adaptive immune response to IAV, and (b) reducing lung injury by reducing E-cadherin shedding, and reducing the accumulation of myeloid leukocytes and their deleterious products in the lungs. Current therapies available to treat serious IAV infections target viral proteins (NA1 and M2) and are suboptimal at limiting IAV-induced morbidity and mortality. MMP-9 small-molecule inhibitors have been developed as novel therapeutics to treat other diseases, but have dose-limiting side effects during chronic dosing that restrict their usefulness in treating chronic diseases (33). However, MMP-9 inhibitors may have a more favorable benefit-risk profile in severe, acute diseases in which dosing would be required for only a relatively short period of time. Our results suggest that MMP-9-selective MMP inhibitors have potential as novel IAV therapeutics targeting the host response to infection. Future clinical trials should be planned to determine whether MMP-9-selective inhibitors have efficacy either when tested alone or in combination with antiviral therapies in limiting IAV-induced morbidity and mortality.

\section{Methods}

Materials. See supplemental material for details.

\section{Approvals}

Human subjects studies. Studies of human subjects were approved by the Institutional Review Boards at Instituto Nacional de Enfermedades Respiratorias Ismael Cosío Villegas and Brigham and Women's Hospital. Written informed consent was received from participants prior to inclusion in the study.

Animal studies. All studies conducted on mice were approved by the Institutional Animal Care and Use Committees at Brigham and Women's Hospital and the Lovelace Respiratory Research Institute. The authors followed ARRIVE guidelines for reporting on animal studies.

Plasma MMP-9 levels in human subjects. Plasma samples were obtained from patients diagnosed with seasonal IAV and pandemic H1N1. These study subjects have been described in detail (67). Samples were collected during the first 2 weeks of onset symptoms following seasonal IAV infection, or during the first 30 days after admission of subjects to the intensive care unit following H1N1 infection. Plasma MMP-9 levels were measured in duplicate using an ELISA (R\&D Systems).

Mice

Colonies of WT and Mmp-9-/- mice, both in a pure C57BL/ 6 background, were housed in a pathogen-free barrier facility under identical conditions (see supplemental material). $\mathrm{Mmp}-\mathrm{9}^{-/-}$mice (backcrossed to the C57BL/6 strain for 10 generations) were generated as described previously (68). The genotypes of the mice were confirmed using PCR-based protocols performed on genomic DNA extracted from tail biopsies.

\section{Generation of $\mathrm{Mmp}-9 \mathrm{BM}$ chimeras}

Mmp-9 BM-chimeric mice were generated by irradiating 8- to 10 -week-old WT and $M m p-9^{-/-}$recipients with 900 cGy using a cesium source and BM isolated from WT or $\mathrm{Mmp}^{-9^{-/-}}$mice delivered by tail vein injection $(69,70)$. BM was allowed to engraft for 10 weeks before mice were infected with H1N1 IAV. Four groups of Mmp- $9^{-/-}$BM chimeras were generated by transplanting (a) WT BM into WT recipients $(n=11)$, (b) WT BM into $M m p-9^{-/-}$recipients $(n=13)$, (c) $M m p-9^{-/-} \mathrm{BM}$ into WT recipients $(n=10)$, and (d) Mmp- $9^{-/-} \mathrm{BM}$ into Mmp- $9^{-1-}$ recipients $(n=5)$.

\section{Virus stock}

The pandemic IAV strain, influenza A/California/07/2009 H1N1 (H1N1), was obtained from the Centers for Disease Control, propagated in Madin-Darby canine kidney (MDCK) cells, and used to infect mice. Titers of stock were measured using a standard plaque assay on 6-well plates of confluent monolayers of MDCK cells (71). See supplemental material for details.

\section{IAV infection of mice}

Adult age- and sex-matched male and female WT and $M m p-9^{-1-}$ mice were randomly assigned to experimental groups by an individual who was not involved in the conduct of the animal studies. Two different inocula 
of $\mathrm{H} 1 \mathrm{~N} 1$ were used. $\mathrm{An} \mathrm{LD}_{80}$ inoculum (10,000 PFU), resulting in 80\% mortality in WT mice, was used to evaluate survival and body weight loss. An $\mathrm{LD}_{20}$ inoculum (1,000 PFU) was used to evaluate lung viral titers, and lung inflammation and injury readouts. Isoflurane-anesthetized mice were inoculated by the intranasal route with H1N1 in endotoxin-free PBS or PBS alone ( $25 \mu 1$ in each nostril). Survival was monitored twice daily, and body weight was measured daily. Mice exhibiting loss of body weight greater than $20 \%$ of baseline or any other significant clinical signs of impending mortality were humanely euthanized.

\section{Murine serum Mmp-9 levels}

Blood samples were obtained from WT mice using cardiac puncture. Blood was allowed to clot on ice, centrifuged (at $500 \mathrm{~g}$ for 5 minutes), and serum was stored at $-80^{\circ} \mathrm{C}$. MMP-9 serum levels were quantified using an ELISA (Cusabio).

Lung levels of Mmp-9, mediators of inflammation, and IFNs

Murine lungs were homogenized in $1 \mathrm{ml}$ of PBS containing $0.5 \%$ Triton X-100, $1 \mathrm{mM}$ phenyl methyl sulfonyl fluoride, $1 \mathrm{mM}$ 1,10-phenanthroline, and Mammalian Protease Inhibitor Cocktail (Sigma-Aldrich). ELISA kits were used to measure murine Mmp-9, I1-1 $\beta$, Il-6, Il-10, Ccl-2, Ccl-5, Tnf- $\alpha$, Tgf- $\beta$ (R\&D Systems), G-csf, Ccl-3, Cxcl-2, Cxcl-1 (PeproTech), Ifn- $\alpha$, Ifn- $\gamma$ (Affymetrix/eBioscience), and Ifn- $\beta$ (PBL Assay Science). Levels of each analyte were normalized to total lung protein level measured using a commercial kit (ThermoFisher Scientific).

\section{Immunofluorescence staining of lung sections for Mmp-9}

Formalin-fixed lung sections from mice were double immunostained with Alexa-488-conjugated antibody for Mmp-9 and with Alexa-546-conjugated antibodies for markers of epithelial cells (pancytokeratin; Sigma-Aldrich, C2562), macrophages (Mac-3; BD Biosciences, 550292), PMN (myeloperoxidase; R\&D Systems, AF3667), or CD8 ${ }^{+}$T cells (CD8; Santa Cruz Biotechnologies, SC-18913), or CD4+ T cells (CD4; Santa Cruz Biotechnologies, SC-1140). Images of the immunostained lung sections were captured and analyzed using a confocal microscope (Olympus Corporation) (72).

\section{Lung viral titers}

Lung viral titers were measured using a modified foci determination assay on 96-well plates of confluent MDCK cells using an immunostaining procedure. The number of viral RNA copies was also measured using qRT-PCR $(73,74)$ on RNA extracted from H1N1-infected lung tissue. See supplemental material for details.

\section{BAL leukocyte counts}

BAL was performed on mice using eight $0.5-\mathrm{ml}$ aliquots of PBS (70). Leukocytes were counted in BAL samples, differential counts were performed on modified Wright's-stained cytocentrifuge preparations, and absolute numbers of macrophages, PMNs, and lymphocytes were calculated.

\section{Lymphocyte subset enumeration in enzymatic lung digests}

WT and $M m p 9^{-1-}$ mice were infected with an $\mathrm{LD}_{20}$ inoculum of $\mathrm{H} 1 \mathrm{~N} 1$ by the intranasal route and were euthanized on days 3,7 , and 10 p.i. Lung tissue was digested with $1 \mathrm{mg} / \mathrm{ml}$ collagenase D (Sigma-Aldrich) at $37^{\circ} \mathrm{C}$ for 30 minutes. Samples were passed through a $70-\mu \mathrm{m}$ strainer and the resulting single-cell suspension was washed twice with cold PBS. Cell viability was quantified with trypan blue dye. Surface staining was performed for 20 minutes at $4^{\circ} \mathrm{C}$ in PBS containing $2 \%$ fetal calf serum (FCS). Intracellular staining for Foxp3 was performed using the Foxp3 Staining Buffer Set (eBioscience). Staining of cells with different markers of leukocytes is described in the supplemental material.

\section{BAL lymphocyte subset counts}

WT and $M m p^{-9^{--}}$mice were infected with an $\mathrm{LD}_{20}$ inoculum of $\mathrm{H} 1 \mathrm{~N} 1$ by the intranasal route and euthanized on day 7 p.i. BAL was performed on mice using eight $0.5-\mathrm{ml}$ aliquots of PBS (70). BAL leukocytes were stimulated ex vivo with Cell Activation Cocktail containing brefeldin A (BioLegend) for 5 hours. Cells were stained for surface antigens for 20 minutes at $4^{\circ} \mathrm{C}$ in PBS containing $2 \% \mathrm{FCS}$, and were fixed using the Fixation/Permeabilization buffer set (BD Biosciences) following the manufacturer's instructions. Briefly, single-cell 
suspensions were incubated with PBS with 2\% FCS and antibodies against surface markers or nonimmune control antibodies on ice for 20 minutes, and then washed. The samples were then incubated with $1 \times$ Fixation/ Permeabilization buffer at room temperature for 30 minutes protected from the light, and washed with $1 \times$ Permeabilization/Wash buffer. After washing the cells, they were incubated with antibodies for intracellular proteins (that were directly conjugated to fluorophores), or appropriate nonimmune control antibodies. The cells were washed and analyzed with flow cytometry. After gating on live CD45 cells, lymphocyte populations were identified as follows: $\mathrm{CD}^{+} \mathrm{T}$ cells $\left(\mathrm{CD} 90.2^{+} \mathrm{CD} 4^{+}\right), \mathrm{CD} 8^{+} \mathrm{T}$ cells $\left(\mathrm{CD} 90.2^{+} \mathrm{CD} 8^{+}\right)$, and NK cells $\left(\mathrm{NK} 1.1^{+} \mathrm{CD} 4^{-} \mathrm{CD}^{-}\right)$. Expression of intracellular cytokines was then analyzed with gating based on fluorescence-minus-one controls. Each lymphocyte population was identified as follows: Tnf- $\alpha^{+}$, Ifn- $\gamma^{+}, \mathrm{Il}-17^{+}$, and Tnf- $\alpha^{+}$Ifn- $\gamma^{+}$, using rat anti-IFN- $\gamma$ IgG1 conjugated to Brilliant Violet 421, rat anti-Tnf- $\alpha$ IgG1 conjugated to $\mathrm{PE}$, and rat anti-Il-17 IgG1 conjugated to PE/Cy7. Flow cytometry was performed on a BD LSRFortessa Cell Analyzer and analyzed using FlowJo software (Tree Star, Inc).

\section{Acute lung injury and E-cadherin shedding}

Mice were infected with an $\mathrm{LD}_{20}$ inoculum of $\mathrm{H} 1 \mathrm{~N} 1$ by the intranasal route and were euthanized on days 3-10 p.i. BAL was performed using one $0.5-\mathrm{ml}$ aliquot of PBS. BALF was removed, and albumin was measured in BALF using an ELISA (GenWay Biotech Inc.). Erythrocytes in the cellular fraction were lysed in $0.5 \mathrm{ml}$ of hypotonic lysis buffer, and hemoglobin released was measured using a QuantiChrom hemoglobin Assay Kit (BioAssay Systems). Respiratory mechanics were measured on anesthetized mice 7 days after they were infected with an $\mathrm{LD}_{20}$ inoculum of $\mathrm{H} 1 \mathrm{~N} 1$ by the intranasal route (75). See supplemental material for details. Soluble (shed) E-cadherin was quantified in BALF samples using an ELISA (R\&D Systems).

\section{Infection of differentiated HBECs and measurement of TEER}

HBECs were isolated from human lung tissue obtained from the organ and tissue donation program of the International Institute for the Advancement of Medicine (76). See supplemental material for details. Cells were grown in monolayers, transferred to transwells, and differentiated at an air-liquid interface (ALI) as described in the supplemental material. HBECs were infected with H1N1 at approximately 0.01-0.6 MOI (400-27,000 PFU/transwell). At intervals, apical surfaces were washed 3 times with $200 \mu 1$ of PBS to measure viral titers, as described for murine lung samples. HBECs were infected with H1N1 (MOI 0.1) and TEER (77) was measured as described in the supplemental material.

\section{Antibody-mediated neutralization of MMP-9 secreted by HBECs}

Rabbit anti-MMP-9 polyclonal IgG (ProteinTech) or nonimmune control IgG (R\&D Systems) was added at $0.55 \mu \mathrm{g} / \mathrm{ml}$ to the basolateral media of confluent HBEC cultures grown at an ALI. Cells were infected with $\mathrm{H} 1 \mathrm{~N} 1$ (or treated with PBS) at a MOI of 0.6 and cultures incubated for 1 hour at $37^{\circ} \mathrm{C}$. The inoculum (or PBS) was removed from the cells and replaced with $250 \mu 1$ of anti-MMP-9 or control IgG added to the apical surface (at $1.37 \mu \mathrm{g} / \mathrm{ml}$ ). Viral titers in apical washes were measured using a foci formation assay (see supplemental material).

\section{Gelatin zymography}

HBECs were grown at an ALI and infected with H1N1 (MOI 3) in the upper well. At 24 and 48 hours p.i., basolateral media were harvested and MMP-9 levels were measured using 10\% gelatin zymograms (BioRad Laboratories). The band intensities were quantified using ImageJ software (version 1.50c4, NIH) (78).

\section{Assessment of cleavage of viral proteins by MMP-9}

See supplemental material for details.

\section{Statistics}

Data were analyzed using SigmaPlot version 13 for Windows (Systat Software Incorporated). The Shapiro-Wilk test was used to determine whether the data were normally distributed, and Brown-Forsythe test was used to assess equal variance. Normally distributed data are presented as mean \pm SEM. Data that were not normally distributed are presented as box-and-whisker plots showing medians and 25th and 75th percentiles, and whiskers showing 10th and 90th percentiles. One-way ANOVAs followed by post hoc testing with 2-tailed Student's $t$ tests or Mann-Whitney $U$ tests were performed. ANOVA for repetitive measures 
was used to analyze changes in body weight from baseline values for each animal. Kaplan-Meier curves were constructed based on the number of failed or censored events, and the Gehan-Breslow-Wilcoxon test was used to compare survival curves (79). For the $\mathrm{Mmp}$-9 BM-chimeric mice, the Gehan-Breslow-Wilcoxon test was applied for curves generated for recipient mice having the same genotype. $P<0.05$ was considered statistically significant.

\section{Author contributions}

$\mathrm{CAO}$ and $\mathrm{KSH}$ conceived the project and designed experimental protocols. CAO, KSH, JRQ, XW, PRB, and JT acquired data, and analyzed and interpreted results. AR, FP, PRB, ARA, and IY conducted experiments and acquired data. JZ, LJ, GR, and $\mathrm{CH}$ provided samples and clinical information. $\mathrm{CAO}, \mathrm{KSH}$, and JRQ wrote the manuscript. All authors reviewed and approved the manuscript.

\section{Acknowledgments}

This work was supported by National Institute of Allergy and Infectious Diseases (NIAID) grants AI111475-01 and AI123516; Department of Defense (CDMRP) grant PR152060; National Heart, Lung, and Blood Institute (NHLBI) grants HL111835, HL086814, PO1 HL105339, and P01 HL114501; Flight Attendants Medical Research Institute grant CIA123046; and the Brigham and Women's HospitalLovelace Respiratory Research Institute Consortium.

Addresses correspondence to: Kevin S. Harrod, Department of Anesthesiology and Perioperative Medicine, Division of Molecular and Translational Biomedicine, School of Medicine, University of Alabama-Birmingham, 901 19th St. S, Building BMR II 324 Birmingham, Alabama 35294, USA. Phone: 205.934.1929; Email: kharrod@uab.edu. Or to: Caroline A. Owen, Division of Pulmonary and Critical Care Medicine, Brigham and Women's Hospital and Harvard Medical School, 60 Fenwood Road, Room 3016H, Building for Transformative Medicine. Boston, Massachusetts 02115, USA. Phone: 617.525.5408; Email: cowen@bwh.harvard.edu.

1. Lim BH, Mahmood TA. Influenza A H1N1 2009 (swine flu) and pregnancy. J Obstet Gynaecol India. 2011;61(4):386-393.

2. Tisoncik JR, Korth MJ, Simmons CP, Farrar J, Martin TR, Katze MG. Into the eye of the cytokine storm. Microbiol Mol Biol Rev. 2012;76(1):16-32.

3. Ma J, Dushoff J, Earn DJ. Age-specific mortality risk from pandemic influenza. J Theor Biol. 2011;288:29-34.

4. Williams WW, et al. Surveillance of vaccination coverage among adult populations - United States, 2015. MMWR Surveill Summ. 2017;66(11):1-28.

5. Frasca D, et al. Unique biomarkers for B-cell function predict the serum response to pandemic H1N1 influenza vaccine. Int Immunol. 2012;24(3):175-182.

6. Crespo M, et al. Efficacy of influenza A H1N1/2009 vaccine in hemodialysis and kidney transplant patients. Clin J Am Soc Nephrol. 2011;6(9):2208-2214.

7. Englund JA. Antiviral therapy of influenza. Semin Pediatr Infect Dis. 2002;13(2):120-128.

8. Crusat M, de Jong MD. Neuraminidase inhibitors and their role in avian and pandemic influenza. Antivir Ther (Lond). 2007;12(4 Pt B):593-602.

9. Owen CA. Leukocyte cell surface proteinases: regulation of expression, functions, and mechanisms of surface localization. Int J Biochem Cell Biol. 2008;40(6-7):1246-1272.

10. Chu EK, et al. Induction of the plasminogen activator system by mechanical stimulation of human bronchial epithelial cells Am J Respir Cell Mol Biol. 2006;35(6):628-638.

11. Partridge CA, Phillips PG, Niedbala MJ, Jeffrey JJ. Localization and activation of type IV collagenase/gelatinase at endothelial focal contacts. Am J Physiol. 1997;272(5 Pt 1):L813-L822.

12. Owen CA, Hu Z, Barrick B, Shapiro SD. Inducible expression of tissue inhibitor of metalloproteinases-resistant matrix metalloproteinase-9 on the cell surface of neutrophils. Am J Respir Cell Mol Biol. 2003;29(3 Pt 1):283-294.

13. Owen CA, Campbell EJ. The cell biology of leukocyte-mediated proteolysis. J Leukoc Biol. 1999;65(2):137-150.

14. Rodenburg KW, Kjoller L, Petersen HH, Andreasen PA. Binding of urokinase-type plasminogen activator-plasminogen activator inhibitor-1 complex to the endocytosis receptors alpha2-macroglobulin receptor/low-density lipoprotein receptor-related protein and very-low-density lipoprotein receptor involves basic residues in the inhibitor. Biochem J. 1998;329(Pt 1):55-63.

15. Corry DB, et al. Overlapping and independent contributions of MMP2 and MMP9 to lung allergic inflammatory cell egression through decreased CC chemokines. FASEB J. 2004;18(9):995-997.

16. Kim JH, et al. Inhibition of matrix metalloproteinase-9 prevents neutrophilic inflammation in ventilator-induced lung injury. Am J Physiol Lung Cell Mol Physiol. 2006;291(4):L580-L587.

17. Albaiceta GM, et al. Lack of matrix metalloproteinase-9 worsens ventilator-induced lung injury. Am J Physiol Lung Cell Mol Physiol. 2008;294(3):L535-L543.

18. Vermeer PD, et al. MMP9 modulates tight junction integrity and cell viability in human airway epithelia. Am J Physiol Lung Cell Mol Physiol. 2009;296(5):L751-L762. 
19. Cowden Dahl KD, et al. Matrix metalloproteinase 9 is a mediator of epidermal growth factor-dependent e-cadherin loss in ovarian carcinoma cells. Cancer Res. 2008;68(12):4606-4613.

20. Bergers G, et al. Matrix metalloproteinase-9 triggers the angiogenic switch during carcinogenesis. Nat Cell Biol. 2000;2(10):737-744.

21. Betsuyaku T, Fukuda Y, Parks WC, Shipley JM, Senior RM. Gelatinase B is required for alveolar bronchiolization after intratracheal bleomycin. Am J Pathol. 2000;157(2):525-535.

22. ARDS Definition Task Force, et al. Acute respiratory distress syndrome: the Berlin Definition. JAMA. 2012;307(23):2526-2533.

23. Grabowska MM, Day ML. Soluble E-cadherin: more than a symptom of disease. Front Biosci (Landmark Ed). 2012;17:1948-1964.

24. McGuire JK, Li Q, Parks WC. Matrilysin (matrix metalloproteinase-7) mediates E-cadherin ectodomain shedding in injured lung epithelium. Am J Pathol. 2003;162(6):1831-1843.

25. Owen CA, Campbell EJ. Extracellular proteolysis: new paradigms for an old paradox. J Lab Clin Med. 1999;134(4):341-351.

26. Song J, et al. PROSPER: an integrated feature-based tool for predicting protease substrate cleavage sites. PLoS One. 2012;7(11):e50300.

27. Liu Q, Zhou YH, Yang ZQ. The cytokine storm of severe influenza and development of immunomodulatory therapy. Cell Mol Immunol. 2016;13(1):3-10.

28. Wang S, et al. Influenza virus-cytokine-protease cycle in the pathogenesis of vascular hyperpermeability in severe influenza. J Infect Dis. 2010;202(7):991-1001

29. Kido H, et al. Role of host cellular proteases in the pathogenesis of influenza and influenza-induced multiple organ failure. Biochim Biophys Acta. 2012;1824(1):186-194.

30. Julkunen I, Sareneva T, Pirhonen J, Ronni T, Melén K, Matikainen S. Molecular pathogenesis of influenza A virus infection and virus-induced regulation of cytokine gene expression. Cytokine Growth Factor Rev. 2001;12(2-3):171-180.

31. Lee YH, Huang JH, Chang TH, Yang HC, Wu-Hsieh BA. Mucosa-associated lymphoid tissue lymphoma translocation protein 1 positively modulates matrix metalloproteinase-9 production in alveolar macrophages upon Toll-like receptor 7 signaling and influenza virus infection. Front Immunol. 2017;8:1177.

32. Foronjy RF, Taggart CC, Dabo AJ, Weldon S, Cummins N, Geraghty P. Type-I interferons induce lung protease responses following respiratory syncytial virus infection via RIG-I-like receptors. Mucosal Immunol. 2015;8(1):161-175.

33. Craig VJ, Zhang L, Hagood JS, Owen CA. Matrix metalloproteinases as therapeutic targets for idiopathic pulmonary fibrosis. Am J Respir Cell Mol Biol. 2015;53(5):585-600.

34. Quintero PA, Knolle MD, Cala LF, Zhuang Y, Owen CA. Matrix metalloproteinase-8 inactivates macrophage inflammatory protein-1 alpha to reduce acute lung inflammation and injury in mice. J Immunol. 2010;184(3):1575-1588.

35. Herold S, Becker C, Ridge KM, Budinger GR. Influenza virus-induced lung injury: pathogenesis and implications for treatment Eur Respir J. 2015;45(5):1463-1478.

36. Ivashkiv LB, Donlin LT. Regulation of type I interferon responses. Nat Rev Immunol. 2014;14(1):36-49.

37. Lin FC, Young HA. Interferons: Success in anti-viral immunotherapy. Cytokine Growth Factor Rev. 2014;25(4):369-376.

38. Hall JC, Rosen A. Type I interferons: crucial participants in disease amplification in autoimmunity. Nat Rev Rheumatol. 2010;6(1):40-49.

39. Turner SJ, Olivas E, Gutierrez A, Diaz G, Doherty PC. Disregulated influenza A virus-specific CD8 ${ }^{+} \mathrm{T}$ cell homeostasis in the absence of IFN-gamma signaling. J Immunol. 2007;178(12):7616-7622.

40. Das G, Sheridan S, Janeway CA. The source of early IFN-gamma that plays a role in Th1 priming. J Immunol. 2001;167(4):2004-2010.

41. Liu Y, et al. Uncompromised NK cell activation is essential for virus-specific CTL activity during acute influenza virus infection. Cell Mol Immunol. 2018;15(9):827-837.

42. Hufford MM, Kim TS, Sun J, Braciale TJ. The effector T cell response to influenza infection. Curr Top Microbiol Immunol. 2015;386:423-455.

43. Marchant DJ, et al. A new transcriptional role for matrix metalloproteinase-12 in antiviral immunity. Nat Med. 2014;20(5):493-502

44. Teijaro JR, et al. Endothelial cells are central orchestrators of cytokine amplification during influenza virus infection. Cell. 2011;146(6):980-991.

45. Grommes J, Soehnlein O. Contribution of neutrophils to acute lung injury. Mol Med. 2011;17(3-4):293-307.

46. Gründemann C, et al. Cutting edge: identification of E-cadherin as a ligand for the murine killer cell lectin-like receptor G1. J Immunol. 2006;176(3):1311-1315.

47. Herndler-Brandstetter D, et al. KLRG1 ${ }^{+}$effector CD8 ${ }^{+} \mathrm{T}$ cells lose KLRG1, differentiate into all memory $\mathrm{T}$ cell lineages, and convey enhanced protective immunity. Immunity. 2018;48(4):716-729.e8.

48. Henson SM, Akbar AN. KLRG1--more than a marker for T cell senescence. Age (Dordr). 2009;31(4):285-291.

49. Streeck H, et al. Epithelial adhesion molecules can inhibit HIV-1-specific CD8+ T-cell functions. Blood. 2011;117(19):5112-5122.

50. Van Den Steen PE, Wuyts A, Husson SJ, Proost P, Van Damme J, Opdenakker G. Gelatinase B/MMP-9 and neutrophil collagenase/MMP-8 process the chemokines human GCP-2/CXCL6, ENA-78/CXCL5 and mouse GCP-2/LIX and modulate their physiological activities. Eur J Biochem. 2003;270(18):3739-3749.

51. Hsieh $\mathrm{CH}$, et al. The role of MIP-1 alpha in the development of systemic inflammatory response and organ injury following trauma hemorrhage. J Immunol. 2008;181(4):2806-2812.

52. Perl M, et al. Divergent effects of activated neutrophils on inflammation, Kupffer cell/splenocyte activation, and lung injury following blunt chest trauma. Shock. 2012;37(2):210-218.

53. Wang W, et al. Macrophage micro-RNA-155 promotes lipopolysaccharide-induced acute lung injury in mice and rats. $A m J$ Physiol Lung Cell Mol Physiol. 2016;311(2):L494-L506.

54. Nawijn MC, Hackett TL, Postma DS, van Oosterhout AJ, Heijink IH. E-cadherin: gatekeeper of airway mucosa and allergic sensitization. Trends Immunol. 2011;32(6):248-255.

55. Correia AL, Mori H, Chen EI, Schmitt FC, Bissell MJ. The hemopexin domain of MMP3 is responsible for mammary epithelial invasion and morphogenesis through extracellular interaction with HSP90ß. Genes Dev. 2013;27(7):805-817.

56. Biswas MH, Du C, Zhang C, Straubhaar J, Languino LR, Balaji KC. Protein kinase D1 inhibits cell proliferation through matrix metalloproteinase-2 and matrix metalloproteinase-9 secretion in prostate cancer. Cancer Res. 2010;70(5):2095-2104. 
57. Stencel-Baerenwald JE, Reiss K, Reiter DM, Stehle T, Dermody TS. The sweet spot: defining virus-sialic acid interactions. Nat Rev Microbiol. 2014;12(11):739-749.

58. Klenk HD, Rott R, Orlich M, Blödorn J. Activation of influenza A viruses by trypsin treatment. Virology. 1975;68(2):426-439.

59. Böttcher E, Matrosovich T, Beyerle M, Klenk HD, Garten W, Matrosovich M. Proteolytic activation of influenza viruses by serine proteases TMPRSS2 and HAT from human airway epithelium. J Virol. 2006;80(19):9896-9898.

60. Mitnaul LJ, et al. Balanced hemagglutinin and neuraminidase activities are critical for efficient replication of influenza A virus. J Virol. 2000;74(13):6015-6020.

61. Talmi-Frank D, et al. Extracellular matrix proteolysis by MT1-MMP contributes to influenza-related tissue damage and mortality Cell Host Microbe. 2016;20(4):458-470.

62. Bradley LM, Douglass MF, Chatterjee D, Akira S, Baaten BJ. Matrix metalloprotease 9 mediates neutrophil migration into the airways in response to influenza virus-induced Toll-like receptor signaling. PLoS Pathog. 2012;8(4):e1002641.

63. Tate MD, Deng YM, Jones JE, Anderson GP, Brooks AG, Reading PC. Neutrophils ameliorate lung injury and the development of severe disease during influenza infection. J Immunol. 2009;183(11):7441-7450.

64. Reilly EC, Lambert-Emo K, Topham DJ. The effects of acute neutrophil depletion on resolution of acute influenza infection, establishment of tissue resident memory (TRM), and heterosubtypic immunity. PLoS ONE. 2016;11(10):e0164247.

65. Polverino F, Seys LJ, Bracke KR, Owen CA. B cells in chronic obstructive pulmonary disease: moving to center stage. Am J Physiol Lung Cell Mol Physiol. 2016;311(4):L687-L695.

66. Proost P, Struyf S, Van Damme J, Fiten P, Ugarte-Berzal E, Opdenakker G. Chemokine isoforms and processing in inflammation and immunity. J Autoimmun. 2017;85:45-57.

67. Zúñiga J, et al. Genetic variants associated with severe pneumonia in A/H1N1 influenza infection. Eur Respir J. 2012;39(3):604-610.

68. Betsuyaku T, Shipley JM, Liu Z, Senior RM. Gelatinase B deficiency does not protect against lipopolysaccharide-induced acute lung injury. Chest. 1999;116(1 Suppl):17S-18S.

69. Knolle MD, et al. Adam8 limits the development of allergic airway inflammation in mice. J Immunol. 2013;190(12):6434-6449.

70. Craig VJ, et al. Profibrotic activities for matrix metalloproteinase-8 during bleomycin-mediated lung injury. J Immunol. 2013;190(8):4283-4296

71. Ware LB. Prognostic determinants of acute respiratory distress syndrome in adults: impact on clinical trial design. Crit Care Med. 2005;33(3 Supp1):S217-S222

72. Campbell EJ, Owen CA. The sulfate groups of chondroitin sulfate- and heparan sulfate-containing proteoglycans in neutrophil plasma membranes are novel binding sites for human leukocyte elastase and cathepsin G. J Biol Chem. 2007;282(19):14645-14654.

73. Matrosovich M, Matrosovich T, Garten W, Klenk HD. New low-viscosity overlay medium for viral plaque assays. Virol J. 2006;3:63

74. Jelley-Gibbs DM, Dibble JP, Brown DM, Strutt TM, McKinstry KK, Swain SL. Persistent depots of influenza antigen fail to induce a cytotoxic CD8 T cell response. J Immunol. 2007;178(12):7563-7570.

75. Laucho-Contreras ME, Taylor KL, Mahadeva R, Boukedes SS, Owen CA. Automated measurement of pulmonary emphysema and small airway remodeling in cigarette smoke-exposed mice. $J$ Vis Exp. 2015;95(95):52236.

76. Fulcher ML, Gabriel S, Burns KA, Yankaskas JR, Randell SH. Well-differentiated human airway epithelial cell cultures. Methods Mol Med. 2005;107:183-206.

77. Srinivasan B, Kolli AR, Esch MB, Abaci HE, Shuler ML, Hickman JJ. TEER measurement techniques for in vitro barrier model systems. J Lab Autom. 2015;20(2):107-126.

78. Hu X, Beeton C. Detection of functional matrix metalloproteinases by zymography. J Vis Exp. 2010;(45):2445.

79. Peto R, et al. Design and analysis of randomized clinical trials requiring prolonged observation of each patient. I. Introduction and design. Br J Cancer. 1976;34(6):585-612. 